\title{
The distribution of warm gas in the G327.3-0.6 massive star-forming region
}

\author{
S. Leurini ${ }^{1}$, F. Wyrowski ${ }^{1}$, F. Herpin ${ }^{2,3}$, F. van der $\operatorname{Tak}^{4,5}$, R. Güsten ${ }^{1}$, and E.F. van Dishoeck ${ }^{6,7}$ \\ 1 Max-Planck-Institut für Radioastronomie, Auf dem Hügel 69, 53121 Bonn, Germany \\ e-mail: sleurini@mpifr.de \\ 2 Univ. de Bordeaux, LAB, UMR 5804, 33270 Floirac, France \\ 3 CNRS, LAB, UMR 5804, 33270 Floirac, France \\ ${ }^{4}$ SRON Netherlands Institute for Space Research, PO Box 800, 9700 AV Groningen, The Netherlands \\ 5 Kapteyn Astronomical Institute, University of Groningen, PO Box 800, 9700 AV Groningen, The Netherlands \\ ${ }^{6}$ Leiden Observatory, Leiden University, PO Box 9513, 2300 RA Leiden, The Netherlands \\ Max Planck Institut für Extraterrestrische Physik, Giessenbachstrasse 1, 85748 Garching, Germany
}

Received 4 February 2012 / Accepted 9 November 2012

\begin{abstract}
Aims. Most studies of high-mass star formation focus on massive and/or luminous clumps, but the physical properties of their larger scale environment are poorly known. In this work, we aim at characterising the effects of clustered star formation and feedback of massive stars on the surrounding medium by studying the distribution of warm gas through mid- $J{ }^{12} \mathrm{CO}$ and ${ }^{13} \mathrm{CO}$ observations.

Methods. We present APEX ${ }^{12} \mathrm{CO}(6-5),(7-6),{ }^{13} \mathrm{CO}(6-5),(8-7)$ and $\mathrm{HIFI}{ }^{13} \mathrm{CO}(10-9)$ maps of the star forming region G327.36-0.6 with a linear size of $\sim 3 \mathrm{pc} \times 4 \mathrm{pc}$. We infer the physical properties of the emitting gas on large scales through a local thermodynamic equilibrium analysis, while we apply a more sophisticated large velocity gradient approach on selected positions.

Results. Maps of all lines are dominated in intensity by the photon dominated region around the HII region G327.3-0.5. Mid- $J$ ${ }^{12} \mathrm{CO}$ emission is detected over the whole extent of the maps with excitation temperatures ranging from $20 \mathrm{~K}$ up to $80 \mathrm{~K}$ in the gas around the HiI region, and $\mathrm{H}_{2}$ column densities from few $10^{21} \mathrm{~cm}^{-2}$ in the inter-clump gas to $3 \times 10^{22} \mathrm{~cm}^{-2}$ towards the hot core G327.3-0.6. The warm gas (traced by ${ }^{12}$ and ${ }^{13} \mathrm{CO}(6-5)$ emission) is only a small percentage $(\sim 10 \%)$ of the total gas in the infrared dark cloud, while it reaches values up to $\sim 35 \%$ of the total gas in the ring surrounding the HII region. The ${ }^{12} \mathrm{CO}$ ladders are qualitatively compatible with photon dominated region models for high density gas, but the much weaker than predicted ${ }^{13} \mathrm{CO}$ emission suggests that it comes from a large number of clumps along the line of sight. All lines are detected in the inter-clump gas when averaged over a large region with an equivalent radius of $50^{\prime \prime}(\sim 0.8 \mathrm{pc})$, implying that the mid- $J{ }^{12} \mathrm{CO}$ and ${ }^{13} \mathrm{CO}$ inter-clump emission is due to high density components with low filling factor. Finally, the detection of the ${ }^{13} \mathrm{CO}(10-9)$ line allows to disentangle the effects of gas temperature and gas density on the $\mathrm{CO}$ emission, which are degenerate in the APEX observations alone.
\end{abstract}

Key words. stars: formation - HII regions - ISM: individual objects: G327.3-0.6

\section{Introduction}

The influence of high-mass stars on the interstellar medium is tremendous. During their process of formation, they are sources of powerful, bipolar outflows (e.g., Beuther et al. 2002), their strong ultraviolet and far-ultraviolet radiation fields give rise to bright HII and photon dominated regions (PDRs) and during their whole lifetime powerful stellar winds interact with the surroundings. Finally, their short life ends in a violent supernova explosion, injecting heavy elements into the interstellar medium and possibly triggering further star formation with the accompanying shocks. These are also the type of regions that dominate far-infrared observations of starburst galaxies.

Most studies of massive star formation focus on emission peaks at infrared or submillimetre wavelengths, which correspond to peaks in the temperature and/or mass distribution. The aim of our work is to characterise the effects of clustered star formation and feedback of massive stars on the surrounding medium. We have made APEX maps of three cluster-forming regions (G327.3-0.6, NGC6334 and W51) in mid- $J{ }^{12} \mathrm{CO}((6-5)$ and $(7-6))$ and ${ }^{13} \mathrm{CO}$ transitions $((6-5)$ and $(8-7))$ in order to have a direct measure of the excitation of the warm extended inter-clump gas between dense cores in the cluster (see for example Blitz \& Stark 1986; Stutzki \& Güsten 1990). Our sample of sources was chosen among six nearby cluster-forming clouds mapped in water and in the ${ }^{13} \mathrm{CO}(10-9)$ transition as part of the Water in Star-Forming Regions with Herschel (WISH; van Dishoeck et al. 2011) guaranteed time key program (GT-KP) for the Herschel Space Observatory (Pilbratt et al. 2010).

In this paper, we present the ${ }^{12} \mathrm{CO}$ and ${ }^{13} \mathrm{CO}$ maps of the starforming region G327.3-0.6 at a distance of $3.3 \mathrm{kpc}$ (Urquhart et al. 2011, based on H I absorption). G327.3-0.6 is well suited to study cluster-forming clouds because of its relatively close distance and because several sources in different evolutionary phases coexist in a small region, as found by Wyrowski et al. (2006). Our maps (with a linear extension of $\sim 3 \mathrm{pc} \times 4 \mathrm{pc}$ ) cover the HII region G327.3-0.5 (Goss \& Shaver 1970) associated with a luminous PDR, and an infrared dark cloud (IRDC; Wyrowski et al. 2006) hosting the bright hot core G327.3-0.6 (Gibb et al. 2000) and the extended green object (EGO) candidate G327.30-0.58 (Cyganowski et al. 2008). Extended green objects are identified through their extended $4.5 \mu \mathrm{m}$ emission in 
A\&A 550, A10 (2013)

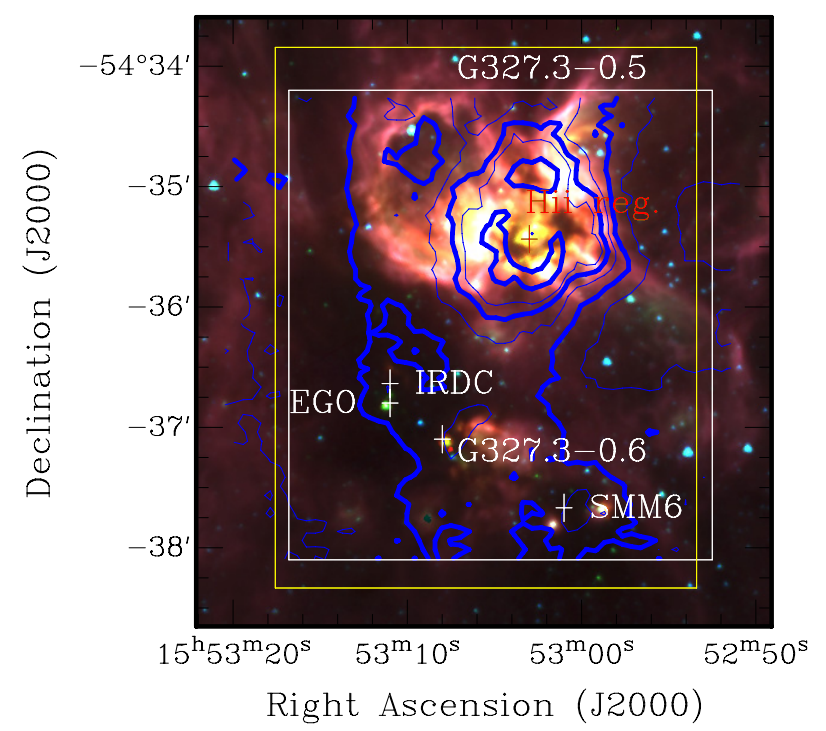

Fig. 1. Spitzer infrared colour image of the region G327.3-0.6 with red representing $8.0 \mu \mathrm{m}$, green $4.5 \mu \mathrm{m}$ and blue $3.6 \mu \mathrm{m}$. The blue contours represent the integrated intensity of the ${ }^{12} \mathrm{CO}(6-5)$ line (thin contours are $15 \%, 45 \%$ and $70 \%$ of the peak emission, thick contours $30 \%, 60 \%$ and $90 \%$ of the peak emission). The sources discussed in this paper (the IRDC, the EGO, the hot core G327.3-0.6, the SMM6 position and the HII region G327.3-0.5) are also marked with crosses. The white and yellow boxes mark the regions mapped with APEX and Herschel, respectively.

the Spitzer IRAC2 band, which is believed to trace outflows from massive young stellar objects (YSOs; Cyganowski et al. 2008).

This paper is organised as follows: in Sect. 2 we present the APEX and Herschel ${ }^{1}$ observations of G327.3-0.6, in Sect. 3 we discuss the morphology and kinematics of the ${ }^{12} \mathrm{CO}$ and ${ }^{13} \mathrm{CO}$ emission, in Sect. 4 we investigate the physical conditions of the emitting gas. Finally, in Sect. 5 we discuss our results and compare to similar observations performed towards low- and high-mass star forming regions. Our results are summarised in Sect. 6.

\section{Observations}

\subsection{APEX telescope}

The $\mathrm{CHAMP}^{+}$(Kasemann et al. 2006; Güsten et al. 2008) dual colour heterodyne array receiver of 7 pixels per frequency channel on the APEX telescope ${ }^{2}$ was used in September 2008 to simultaneously map the star-forming region G327.3-0.6 in the ${ }^{12} \mathrm{CO}(6-5)$ and (7-6) lines and, in a second coverage, the ${ }^{13} \mathrm{CO}(6-5)$ and $(8-7)$ transitions. The region from the hot core in G327.3-006 to the H II region G327.3-0.5 (Fig. 1) was covered with on-the-fly maps of $200^{\prime \prime} \times 240^{\prime \prime}$ spaced by $4^{\prime \prime}$ in declination and right ascension.

We used the fast Fourier transform spectrometer (FFTS, Klein et al. 2006) as backend with two units of fixed bandwidth of $1.5 \mathrm{GHz}$ and 8192 channels per pixel. We used the two IF groups of the FFTS with an offset of $\pm 460 \mathrm{MHz}$ between them. The original resolution of the dataset is $0.3 \mathrm{~km} \mathrm{~s}^{-1}$; the

\footnotetext{
1 Herschel is an ESA space observatory with science instruments provided by European-led Principal Investigator consortia and with important participation from NASA.

2 This publication is based on data acquired with the Atacama Pathfinder Experiment (APEX). APEX is a collaboration between the Max-Planck-Institut fur Radioastronomie, the European Southern Observatory, and the Onsala Space Observatory
}

Table 1. Observational parameters.

\begin{tabular}{lccccc}
\hline \hline Line & $\begin{array}{c}\text { Frequency } \\
(\mathrm{GHz})\end{array}$ & Telescope & $\begin{array}{c}\text { Beam } \\
\left({ }^{\prime \prime}\right)\end{array}$ & $\begin{array}{c}\Delta v \\
\left(\mathrm{~km} \mathrm{~s}^{-1}\right)\end{array}$ & $\begin{array}{c}\mathrm{rms} \\
(\mathrm{K})\end{array}$ \\
\hline $\mathrm{CO}(6-5)$ & 691.473 & APEX & $9 !^{\prime} 0$ & 1.0 & 1.4 \\
$\mathrm{CO}(7-6)$ & 806.652 & APEX & $77^{\prime \prime} 7$ & 1.0 & 4.4 \\
${ }^{13} \mathrm{CO}(6-5)$ & 661.067 & APEX & $9 !^{\prime} 4$ & 1.0 & 1.1 \\
${ }^{13} \mathrm{CO}(8-7)$ & 881.273 & APEX & $7{ }^{\prime \prime} 1$ & 1.0 & 2.8 \\
${ }^{13} \mathrm{CO}(10-9)$ & 1101.350 & Herschel & 19.90 & 1.1 & 0.1 \\
\hline
\end{tabular}

spectra were smoothed to $1 \mathrm{~km} \mathrm{~s}^{-1}$ for a better signal-to-noise ratio.

The observations were performed under good weather conditions with a precipitable water vapour level between 0.5 and $0.7 \mathrm{~mm}$. Typical single side band system temperatures during the observations were around $1600 \mathrm{~K}$ and $5200 \mathrm{~K}$, for the low and high frequency channel respectively. The conversion from antenna temperature units to brightness temperatures was done assuming a forward efficiency of 0.95 for all channels, and a main beam efficiency of 0.48 for the ${ }^{12} \mathrm{CO}$ and ${ }^{13} \mathrm{CO}(6-5)$ observations, 0.45 for the ${ }^{12} \mathrm{CO}(7-6)$ data, and 0.44 for ${ }^{13} \mathrm{CO}(8-7)$, as measured on Jupiter in September $2008^{3}$. The pointing was checked on the continuum of the hot core G327.3-0.6 $\left(\alpha_{\mathrm{J} 2000}=\right.$ $\left.15^{\mathrm{h}} 53^{\mathrm{m}} 07^{\mathrm{s}} .8, \delta_{\mathrm{J} 2000}=-54^{\circ} 36^{\prime} 06^{\prime \prime} .4\right)$. The maps were produced with the XY_MAP task of CLASS904, which convolves the data with a Gaussian of one third of the beam: the final angular resolution is 9.' 4 for the low frequency data, 8.' 1 for the high frequency.

\subsection{Herschel space observatory}

The ${ }^{13} \mathrm{CO}(10-9)$ line (see Table 1 ) was mapped (size $=210^{\prime \prime} \times$ $270^{\prime \prime}$ ) with the HIFI instrument (de Graauw et al. 2010) towards G327.3-0.6 on February, 18th, 2011 (observing day - OD) 645, observing identification number (OBSID) 1342214421. The centre of the map is $\alpha_{\mathrm{J} 2000}=15^{\mathrm{h}} 53^{\mathrm{m}} 05^{\mathrm{s}} .48, \delta_{\mathrm{J} 2000}=$ $-54^{\circ} 36^{\prime} 06^{\prime \prime} 2$. The observations are part of the WISH GT-KP (van Dishoeck et al. 2011). Data were taken simultaneously in $\mathrm{H}$ and $\mathrm{V}$ polarisations using both the acousto-optical Wide-Band Spectrometer (WBS) with $1.1 \mathrm{MHz}$ resolution and the correlator-based high-resolution spectrometer (HRS) with $250 \mathrm{kHz}$ nominal resolution. In this paper we present only the WBS data. We used the on-the-fly mapping mode with Nyquist sampling. HIFI receivers are double sideband with a sideband ratio close to unity. The double side band system temperatures and total integration times are respectively $384 \mathrm{~K}$ and $3482 \mathrm{~s}$. The rms noise level at $1 \mathrm{~km} \mathrm{~s}^{-1}$ spectral resolution is $\sim 0.1 \mathrm{~K}$. Calibration of the raw data onto $T_{\mathrm{A}}$ scale was performed by the in-orbit system (Roelfsema et al. 2012); conversion to $T_{\mathrm{mb}}$ was done with a beam efficiency of 0.74 and a forward efficiency of 0.96 . The flux scale accuracy is estimated to be around $15 \%$ for band 3. Data calibration was performed in the Herschel Interactive Processing Environment (HIPE, Ott 2010) version 6.0. Further analysis was done within the CLASS90 package. After inspection, data from the two polarisations were averaged together.

The original angular resolution of the data is $199^{\prime} 0$. The final maps were produced with the XY_MAP task of CLASS90 and have an angular resolution of $21^{\prime \prime} 1$.

\footnotetext{
http://www .mpifr-bonn .mpg.de/div/submmtech/ heterodyne/champplus/champmain.html

4 http://www.iram. fr/IRAMFR/GILDAS/
} 


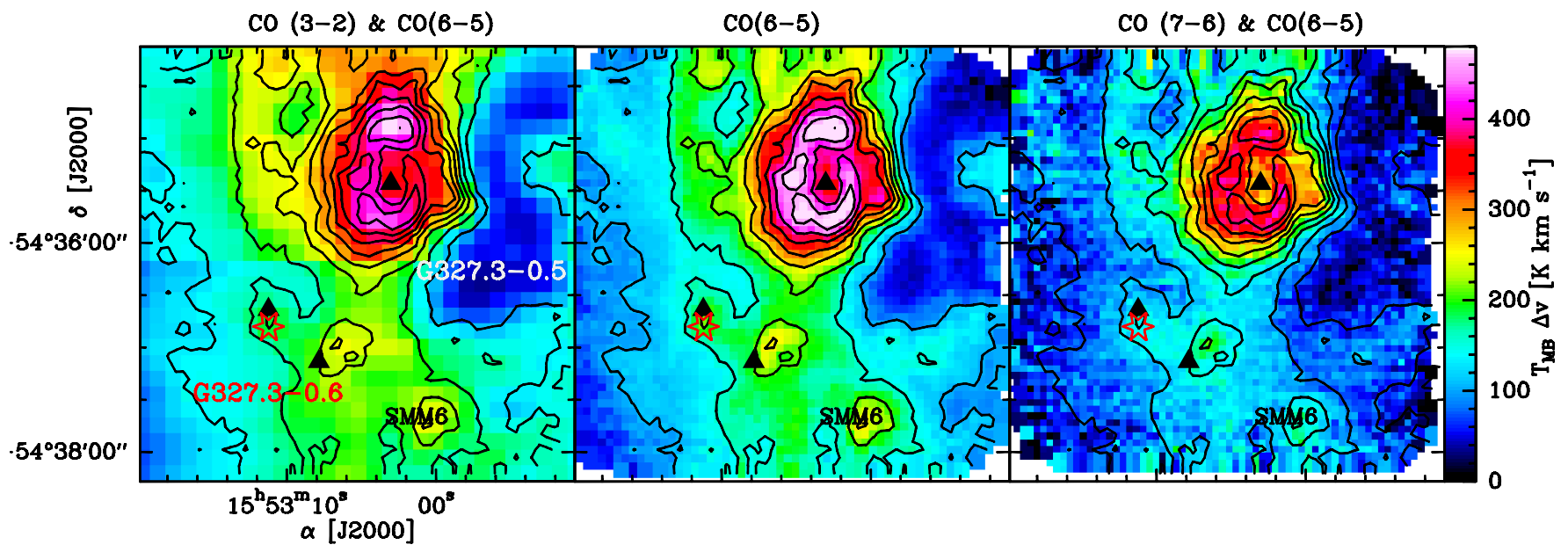

Fig. 2. Maps of the integrated intensity of the ${ }^{12} \mathrm{CO}(3-2),(6-5)$ and (7-6) lines in the velocity range $v_{\text {LSR }}=[-54,-40] \mathrm{km} \mathrm{s}^{-1}$ (color scale). Solid contours are the integrated intensity of the ${ }^{12} \mathrm{CO}(6-5)$ line from $20 \%$ of the peak emission in steps of $10 \%$. In each panel, the positions analysed in Sect. 4.2 (the hot core, the IRDC position, and the centre of the HII region) are marked with black triangles. The red star labels the position of the EGO. SMM6 (left panel) is one of the submillimetre sources detected by Minier et al. (2009).

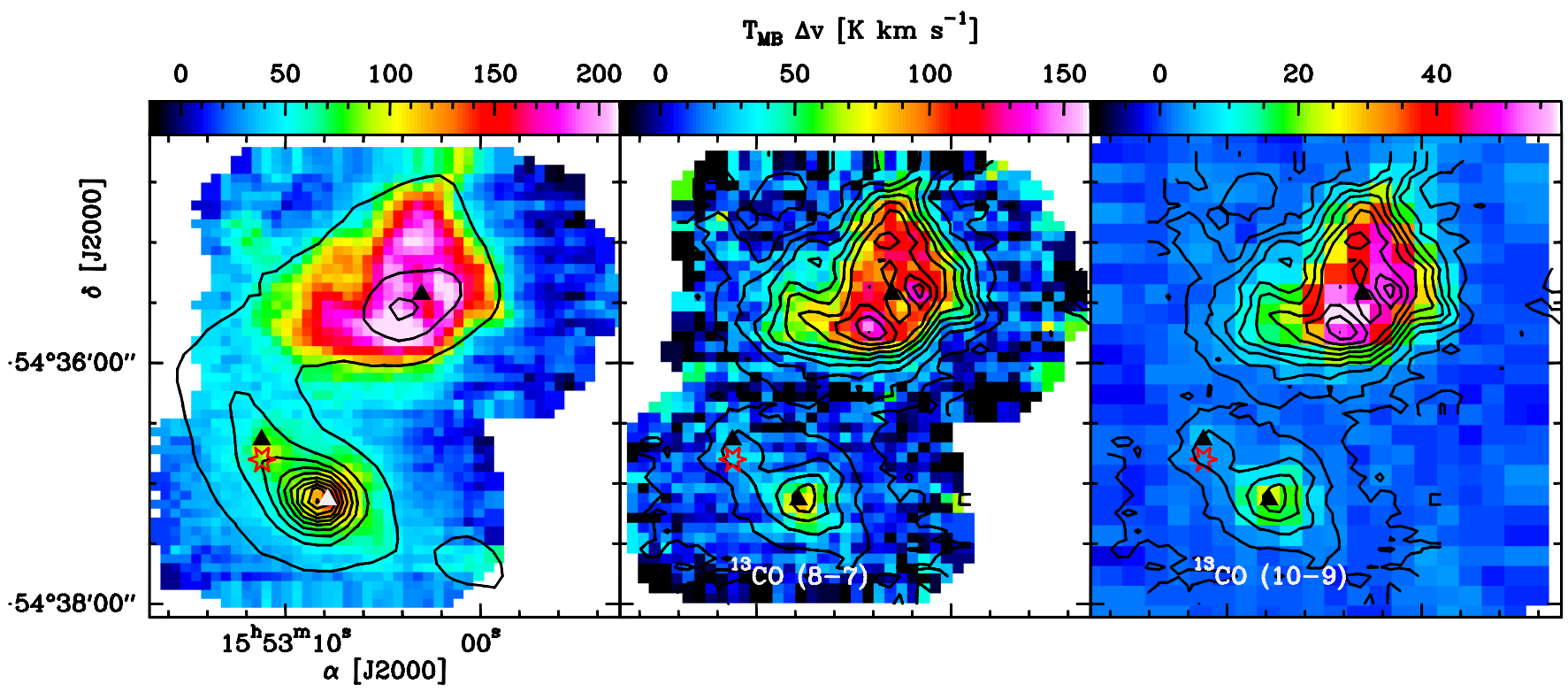

Fig. 3. Maps of the integrated intensity of the ${ }^{13} \mathrm{CO}(6-5),(8-7)$ and (10-9) transitions in the velocity range $v_{\mathrm{LSR}}=[-54,-40] \mathrm{km} \mathrm{s}{ }^{-1}$ (colour scale). The solid contours in the left panel represent the LABOCA continuum emission at $870 \mu \mathrm{m}$ from 5\% of the peak emission in steps of $10 \%$ (Schuller et al. 2009). In the middle and right panels, the solid contours are the integrated intensity of the ${ }^{13} \mathrm{CO}(6-5)$ from $20 \%$ of the peak emission in steps of 10\%. In each panel, the positions analysed in Sect. 4.2 (the hot core, the IRDC position, and the centre of the HII region) are marked with black triangles (except in the left panel, where the hot core is shown by a white triangle). The red star labels the position of the EGO.

\section{Observational results}

\subsection{Morphology}

Figure 1 shows the ${ }^{12} \mathrm{CO}(6-5)$ integrated intensity map overlaid on the composite image of the IRAC Spitzer 3.6, 4.5 and $8.0 \mu \mathrm{m}$ bands of the region. The ${ }^{12} \mathrm{CO}$ emission traces the distribution of the $8.0 \mu \mathrm{m}$ emission, but it is also associated with the infrared dark cloud found on the east of the hot core. The EGO candidate G327.30-0.58 identified by Cyganowski et al. (2008) and clearly visible in Fig. 1, is also detected in the ${ }^{12} \mathrm{CO}$ data as secondary peak of emission (Fig. 2). The map of the integrated intensities of the ${ }^{12} \mathrm{CO}(7-6)$ line is also presented in Fig. 2 together with the integrated intensity of the ${ }^{12} \mathrm{CO}(3-2)$ line from Wyrowski et al. (2006). Figure 3 shows the distribution of the
${ }^{13} \mathrm{CO}(6-5),(8-7)$ and (10-9) emissions. The accuracy of the relative pointings was checked on the hot core G327.3-0.6. For this purpose, we derived integrated intensity maps of lines detected only towards this position, which are close in frequency to the ${ }^{12} \mathrm{CO}(6-5),{ }^{13} \mathrm{CO}(6-5)$ and ${ }^{13} \mathrm{CO}(8-7)$ transitions, and therefore were observed simultaneously to the current dataset. From these data, we infer a position for the hot core in agreement with interferometric measurements at $3 \mathrm{~mm}$ (Wyrowski et al. 2008, and Table 2) within $\sim 1$ '. 5 .

All observed ${ }^{12} \mathrm{CO}$ transitions trace the HII region G327.3-0.5 as well as the infrared dark cloud which hosts the hot core G327.3-0.6. Moreover, the ${ }^{12} \mathrm{CO}(6-5),(7-6)$ and ${ }^{13} \mathrm{CO}(6-5)$ lines show extended emission along a ridge running approximately $\mathrm{N}-\mathrm{S}$ that matches very well with the distribution of the $\mathrm{CO}(3-2)$ transition. The hourglass shape hole to the west 
Table 2. Coordinates of the main sources in the G327.3-0.6 massive star-forming region.

\begin{tabular}{lcc}
\hline \hline Source & $\alpha_{\text {J2000 }}$ & $\delta_{\text {J2000 }}$ \\
\hline SMM6 $^{a}$ & $15: 53: 00.9$ & $-54: 37: 40.0$ \\
hot core & $15: 53: 07.8$ & $-54: 37: 06.4$ \\
EGO $^{c}$ & $15: 53: 11.2$ & $-54: 36: 48.0$ \\
HII $^{d}$ & $15: 53: 03.0$ & $-54: 35: 25.6$ \\
\hline
\end{tabular}

Notes. ${ }^{(a)}$ Minier et al. (2009). ${ }^{(b)}$ Wyrowski et al. (2008). ${ }^{\left({ }^{(c)}\right.}$ Cyganowski et al. (2008). ${ }^{(d)}$ Peak of the centimetre continuum emission from ATCA archival data at $2.3 \mathrm{GHz}$, project number $\mathrm{C} 772$.

of the HII region G327.3-0.5 where the ${ }^{12} \mathrm{CO}(3-2)$ emission is strongly reduced (see Wyrowski et al. 2006) is seen also in the ${ }^{12} \mathrm{CO}(6-5)$ and $(7-6)$ lines which, although much weaker than in the rest of the map, are still detected at this position.

All transitions peak towards the HII region G327.3-0.5 where the main isotopologue lines have intensities up to $60-65 \mathrm{~K}$. The integrated intensities of the CO isotopologues show a distribution along a ring-like structure around the peak of the $\mathrm{cm}$ continuum emission (Goss \& Shaver 1970). The centre of the ring also coincides with the massive young stellar object number 87 identified in the near-infrared by Moisés et al. (2011). Since the ring is detected also in high- $J$ transitions of ${ }^{13} \mathrm{CO}$, it is plausible that this morphology is true and not due to optical depth effects. This structure likely coincides with the limb brightening of the hot surface of a PDR around G327.3-0.5 and could trace an expanding shell. We will investigate this scenario in Sect. 3.2.

The hot core G327.3-0.6 shows up as a secondary peak in the integrated intensity maps of the ${ }^{13} \mathrm{CO}$ transitions, while the main $\mathrm{CO}$ isotopologue peaks to its north-west, probably because of optical depth effects. Strong self-absorption profiles are indeed detected in all ${ }^{12} \mathrm{CO}$ lines towards the hot core (see Sect. 3.2). The submillimetre source SMM6 (seen in the continuum emission at $450 \mu \mathrm{m}$ by Minier et al. 2009) is detected as a peak of emission in all integrated intensity maps of ${ }^{12} \mathrm{CO}$ and in ${ }^{13} \mathrm{CO}(6-5)$, although at the edge of the mapped region. The other submillimetre sources are also marked in Figs. 2. The EGO candidate G327.30-0.58 is also detected in the ${ }^{13} \mathrm{CO}(6-5)$ map (Fig. 3). The ${ }^{13} \mathrm{CO}(6-5)$ traces the whole IRDC and not only the active site of star formation where the EGO is detected. The continuum emission due to dust (seen for example at $870 \mu \mathrm{m}$ in Fig. 3) follows the distribution of the ${ }^{13} \mathrm{CO}$ lines.

In Fig. 4 we show the ratio of the integrated intensity of the ${ }^{12} \mathrm{CO}(6-5)$ transition (convolved to the $18^{\prime \prime}$ resolution of the ${ }^{12} \mathrm{CO}(3-2)$ data) to that of the ${ }^{12} \mathrm{CO}(3-2)$ line. This ratio ranges between 0.3 and 1.8; it has values slightly larger than one towards the HII region (1.2 at its centre), while it is about unity towards the hot core. The peak is found south-west of the HII region G327.3-0.5, where both lines are detected with a high confidence level. However, these results could be biased by the strong self absorption in both ${ }^{12} \mathrm{CO}$ lines (see Sect. 3.2). For this reason, we computed the ratio between the two transitions in four velocity ranges to cross-check the results of Fig. 4. The inferred values, however, do not change significantly.

\subsection{Line profiles and velocity field}

The widespread ${ }^{12} \mathrm{CO}(6-5)$ emission shows line profiles with a typical width of $\sim 8 \mathrm{~km} \mathrm{~s}^{-1}$ in the gas between G327.3-0.5

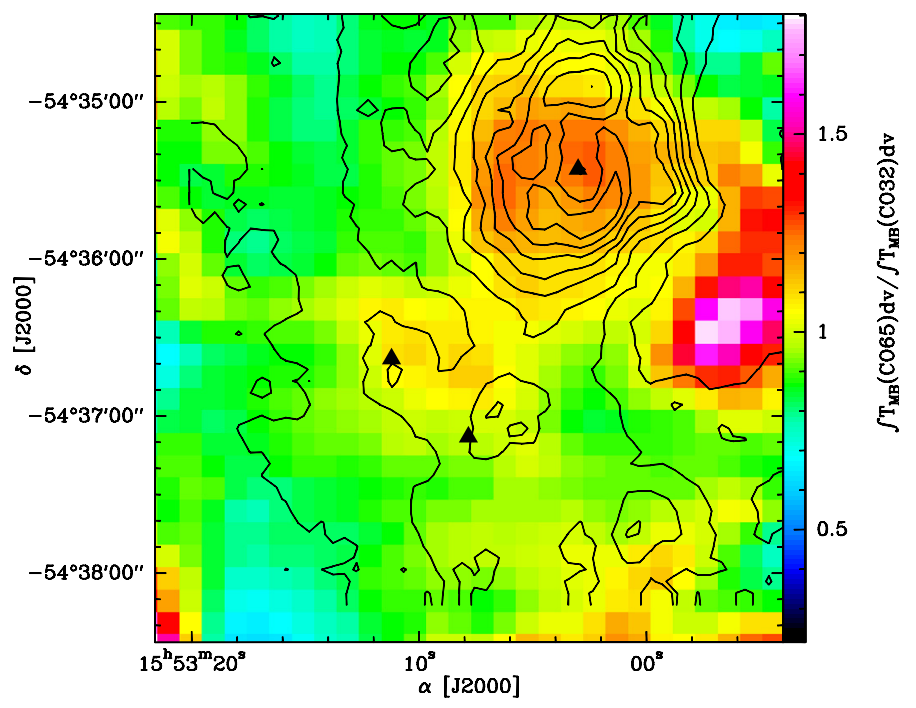

Fig. 4. Distribution of the line ratio of the ${ }^{12} \mathrm{CO}(6-5)$ transition to the ${ }^{12} \mathrm{CO}(3-2)$ line. Solid contours show the ${ }^{12} \mathrm{CO}(6-5)$ integrated intensity in the velocity range $\mathrm{v}_{\mathrm{LSR}}=[-54,-40] \mathrm{km} \mathrm{s}^{-1}$ from $20 \%$ of the peak value in steps of $10 \%$. The black triangles are as in Fig. 2.

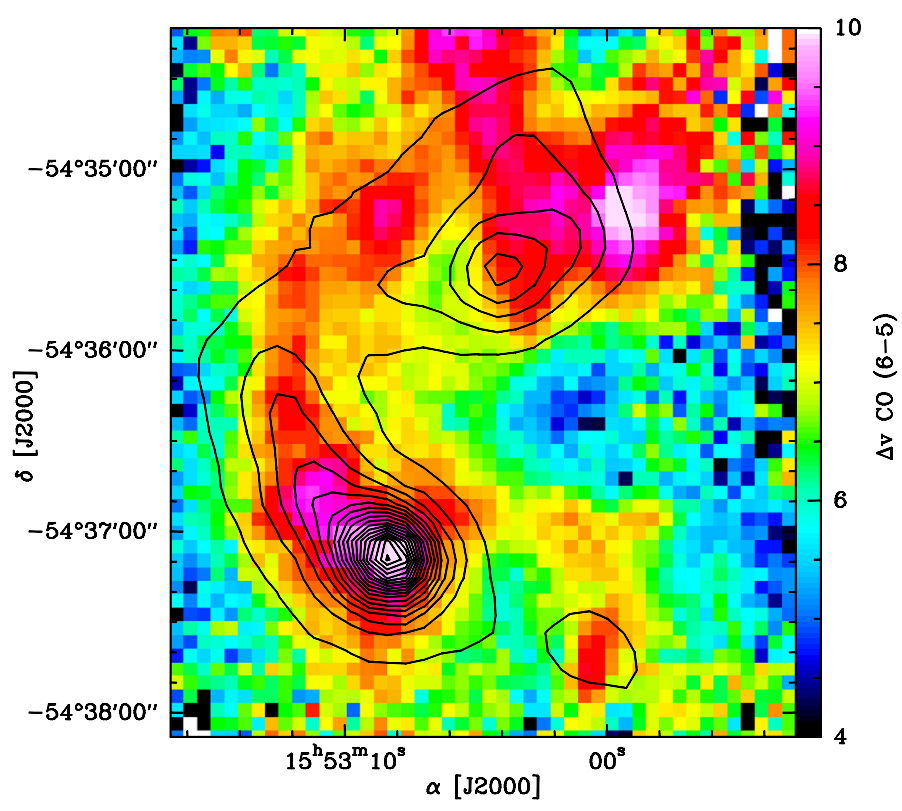

Fig. 5. Distribution of the second moment of the ${ }^{12} \mathrm{CO}(6-5)$ line. The black solid contours represent the LABOCA continuum emission at $870 \mu \mathrm{m}$ from $5 \%$ of the peak emission in steps of $5 \%$. The peak of the continuum emission corresponds to the hot core position.

and the infrared dark cloud. Broader profiles are detected in the infrared dark cloud and in the northern part of the HII region G327.3-0.5. Figure 5 shows the distribution of the line width of the ${ }^{12} \mathrm{CO}(6-5)$ transition: the ${ }^{12} \mathrm{CO}(6-5)$ line width follows an arc-like structure that connects the HII region G327.3-0.5 to the infrared dark cloud where the hot core is. Interestingly, the same morphology is seen in the LABOCA map of the region (Schuller et al. 2009). Line widths are similar for all ${ }^{12} \mathrm{CO}$ lines, while they are consistently narrower in the ${ }^{13} \mathrm{CO}$ transitions.

Representative spectra of all ${ }^{12} \mathrm{CO}$ transitions analysed in this study are presented in Fig. 6 towards the hot core, the IRDC position $\left(\left(30^{\prime \prime}, 30^{\prime \prime}\right)\right.$ from the centre of the APEX maps, see Sect. 4.2 and Figs. 2, 3) and the peak of the cm continuum emission in G327.3-0.5. Spectra of the ${ }^{13} \mathrm{CO}$ transitions 


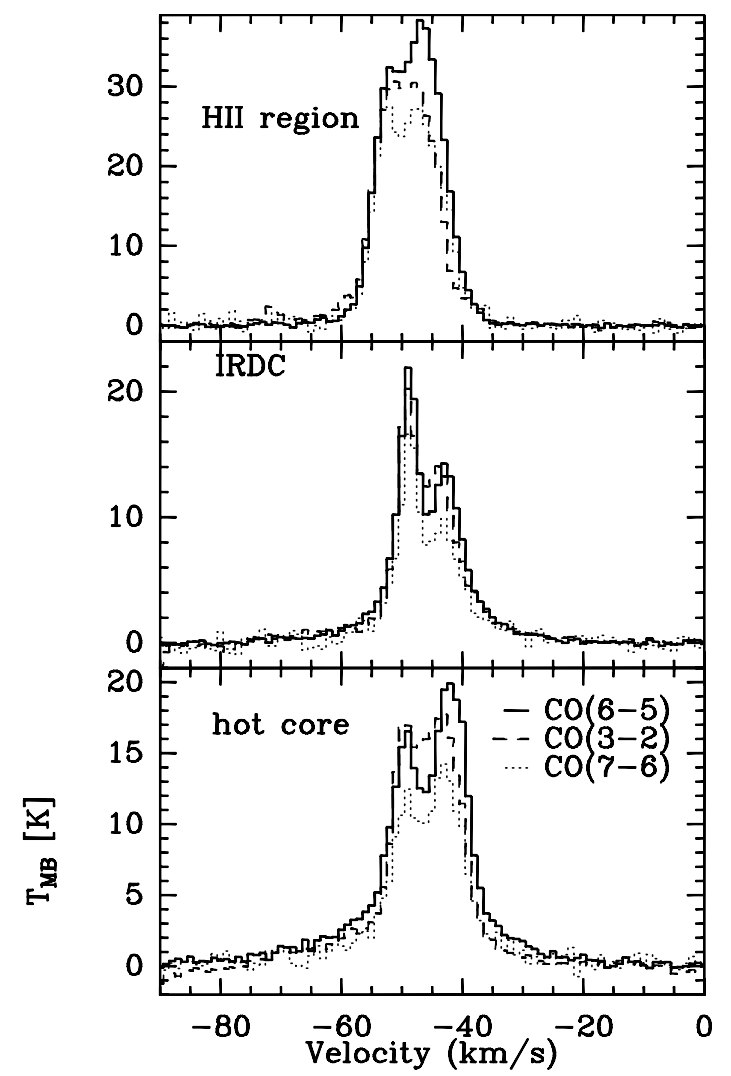

Fig. 6. Mean spectra over a beam of $18^{\prime \prime}$ (to match the resolution of the ${ }^{12} \mathrm{CO}(3-2)$ data) of the ${ }^{12} \mathrm{CO}$ isotopologue transitions analysed in the paper. The top panel shows spectra at the centre of G327.3-0.5, the middle panel spectra at the IRDC position, the bottom panel spectra at the hot core position.

are shown in Fig. 7. Red- and blue-shifted wings are detected in the ${ }^{12} \mathrm{CO}$ lines in a velocity range between -71 and $-24 \mathrm{~km} \mathrm{~s}^{-1}$ (in ${ }^{12} \mathrm{CO}(6-5)$ ) towards G327.3-0.6 probably due to outflow motions. However, no sign of bipolar outflows is found when inspecting the integrated intensity maps of the blueand red-shifted wings nor in position-velocity diagrams (Fig. 8). Moreover, very similar broad lines are detected along the whole extent of the infrared dark cloud, as shown in the top panel of Fig. 8. At the IRDC position, the wings in ${ }^{12} \mathrm{CO}(6-5)$ range from -60 to $-31 \mathrm{~km} \mathrm{~s}^{-1}$. All main isotopologue transitions analysed in this paper are affected by self-absorption (see Fig. 6 for reference spectra towards the hot core, the IRDC position and the HII region); moreover, even the ${ }^{13} \mathrm{CO}(6-5)$ line shows weak evidence of self-absorbed profile towards the hot core. Figure 9 shows the ${ }^{12} \mathrm{CO}(7-6)$ spectra overlaid on the continuum emission at $870 \mu \mathrm{m}$ : the self-reversed profile is spread over a large area and seems to follow the thermal dust continuum emission.

Finally, the velocity field of the ${ }^{12} \mathrm{CO}$ transitions may help us to understand the nature of the ring detected towards the HII region G327.3-0.5. We therefore used the task KSHELL built in the visualisation software package KARMA (Gooch 1996). KSHELL computes an average brightness temperature on annuli about a user defined centre. A spherically symmetric expanding shell will look like a half ellipse in a $(r-v)$ diagram with the axis in the $v$ direction twice the expansion velocity. Figure 10 shows the resulting $(r-v)$ diagram obtained with the ${ }^{12} \mathrm{CO}(6-5)$ data cube using the peak of the $\mathrm{cm}$ continuum emission as centre. The emission does not follow a perfect spherical shell. This is likely due to inhomogeneities in the distribution of the gas, as

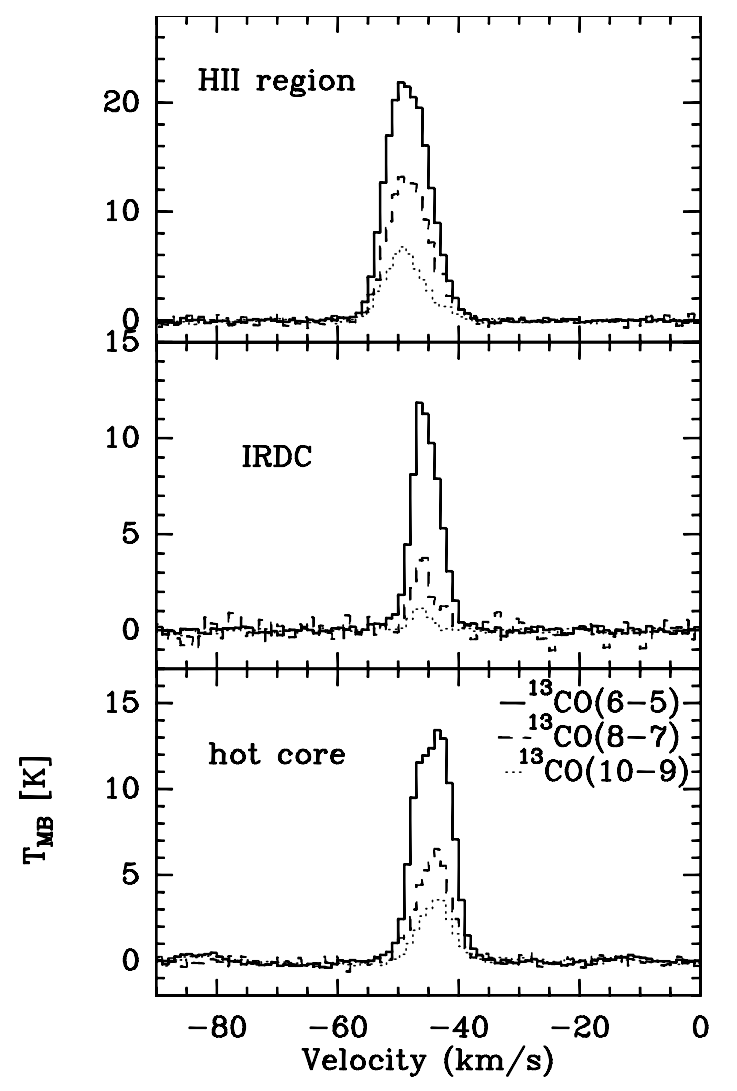

Fig. 7. Mean spectra over a beam of 21 ". 1 (to match the resolution of the ${ }^{13} \mathrm{CO}(10-9)$ data) of the ${ }^{13} \mathrm{CO}$ isotopologue transitions. The selected positions are those discussed in Sect. 4.2.

already seen in Fig. 11 where the distribution of the optical depth of ${ }^{13} \mathrm{CO}$ is not symmetric.

\section{Physical conditions of the warm gas}

\subsection{LTE analysis}

From the line ratio of the ${ }^{12} \mathrm{CO}(6-5)$ to ${ }^{13} \mathrm{CO}(6-5)$ transitions we can derive the optical depth of the ${ }^{12} \mathrm{CO}(6-5)$ line emission, which can be then used to infer the excitation temperature of the line and the column density of ${ }^{12} \mathrm{CO}$ in the region.

The line intensity in a given velocity channel of a given transition is

$T_{\mathrm{L}}=\eta \times\left[F_{v}\left(T_{\mathrm{ex}}\right)-F_{v}\left(T_{\mathrm{cbg}}\right)\right] \times\left(1-\mathrm{e}^{-\tau_{v}}\right)$

where $\eta$ is the beam filling factor (assumed to be 1 in the following analysis $), F_{v}=h v / k \times[\exp (\mathrm{h} v / \mathrm{kT})-1]^{-1}, T_{\mathrm{cbg}}=$ $2.7 \mathrm{~K}$, and $\tau_{v}$ is the optical depth. Under the local thermodynamic equilibrium (LTE) assumption, $T_{\mathrm{ex}}$ is assumed to be equal to the kinetic temperature of the gas and equal for all transitions. In the following analysis, we study the peak intensities of the ${ }^{12} \mathrm{CO}(6-5)$ and ${ }^{13} \mathrm{CO}(6-5)$ lines, and include only the cosmic background as background radiation and neglect, for example, any contribution from infrared dust emission since we do not have any map of the distribution of the dust temperature. This most likely affects only our estimates at the hot core position and possibly towards the HII region G327.3-0.5 where SABOCA continuum emission at $350 \mu \mathrm{m}$ is also detected (Wyrowski et al., in prep.). For an appropriate analysis of the emission from the hot core, see Rolffs et al. (2011). 


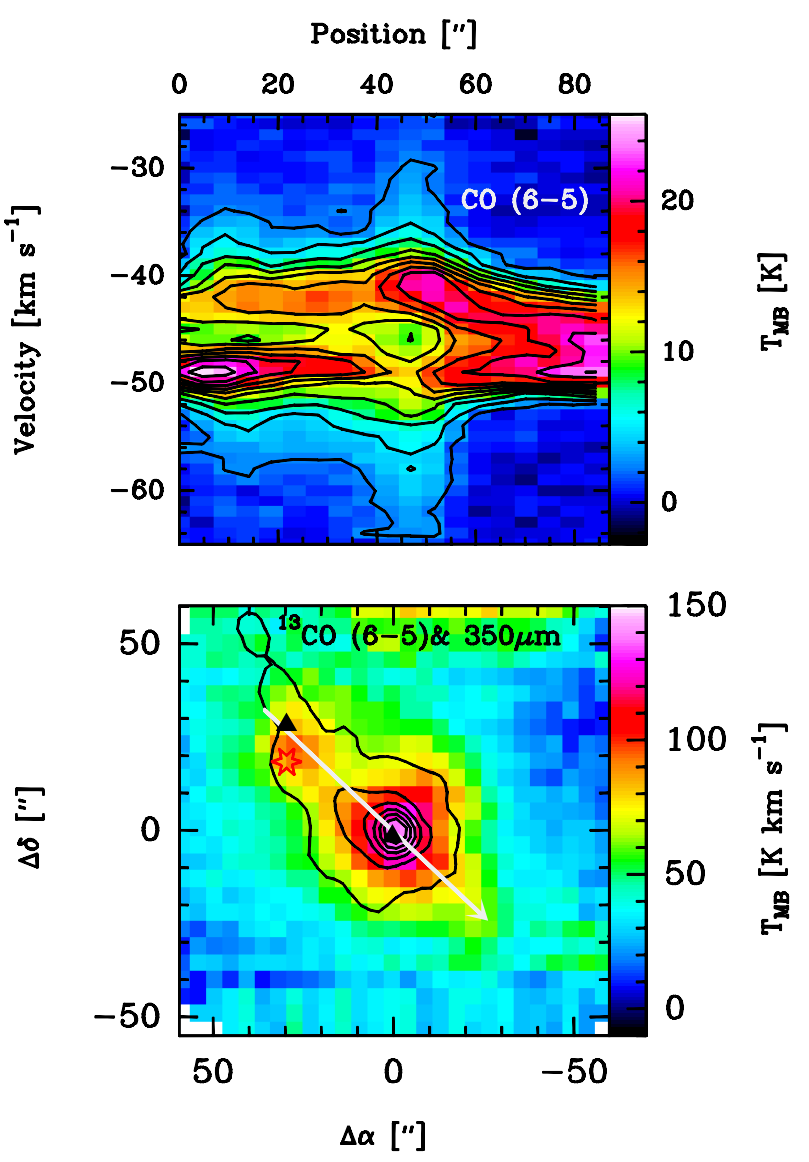

Fig. 8. Top panel: color scale and contours show the $P-V$ diagram of the $\mathrm{CO}(6-5)$ transition computed along the cut indicated by the white arrow in the bottom panel. Offset positions increase along the direction of the arrow shown in the bottom panel. Bottom panel: distribution of the integrated intensity of the ${ }^{13} \mathrm{CO}(6-5)$ transition towards the hot core G327.3-0.6. Solid contours show the continuum emission at $350 \mu \mathrm{m}$ (Wyrowski et al., in prep.) from $3 \sigma$ in steps of $10 \sigma(\sigma \sim 3 \mathrm{Jy} /$ beam). Symbols are as in Fig. 2.

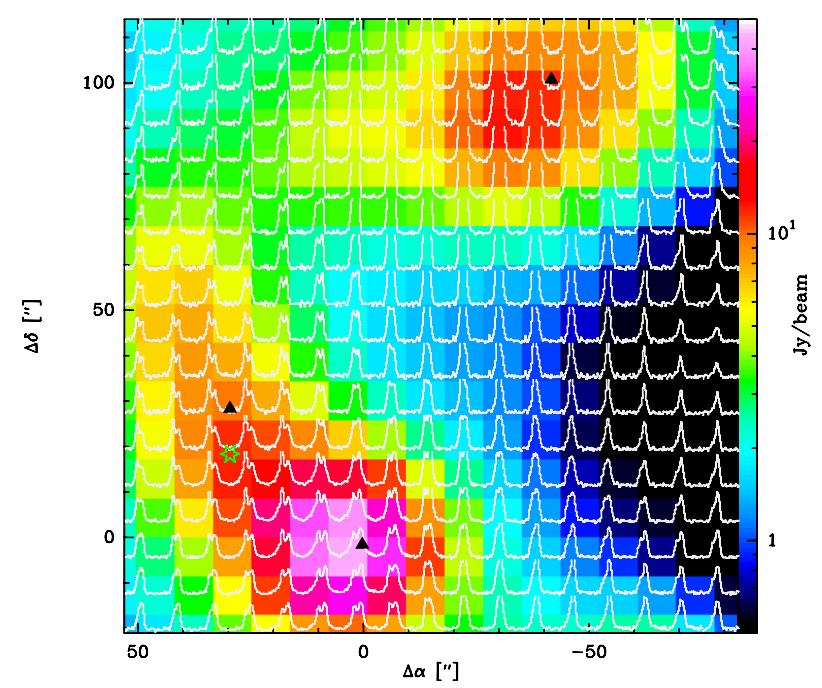

Fig. 9. Map of the ${ }^{12} \mathrm{CO}(7-6)$ line overlaid on the continuum emission at $870 \mu \mathrm{m}$ from LABOCA. The velocity axis ranges from -60 to $-25 \mathrm{~km} \mathrm{~s}^{-1}$, the temperature axis from -1 to $15 \mathrm{~K}$. The ${ }^{12} \mathrm{CO}(7-6)$ data were smoothed to a resolution of $18^{\prime \prime}$ to match the resolution of the ${ }^{12} \mathrm{CO}(3-2)$ data and of the LABOCA emission. The centre of the map is that of the APEX data (see Sect. 2.1). The triangles and the green star are as in Fig. 2.

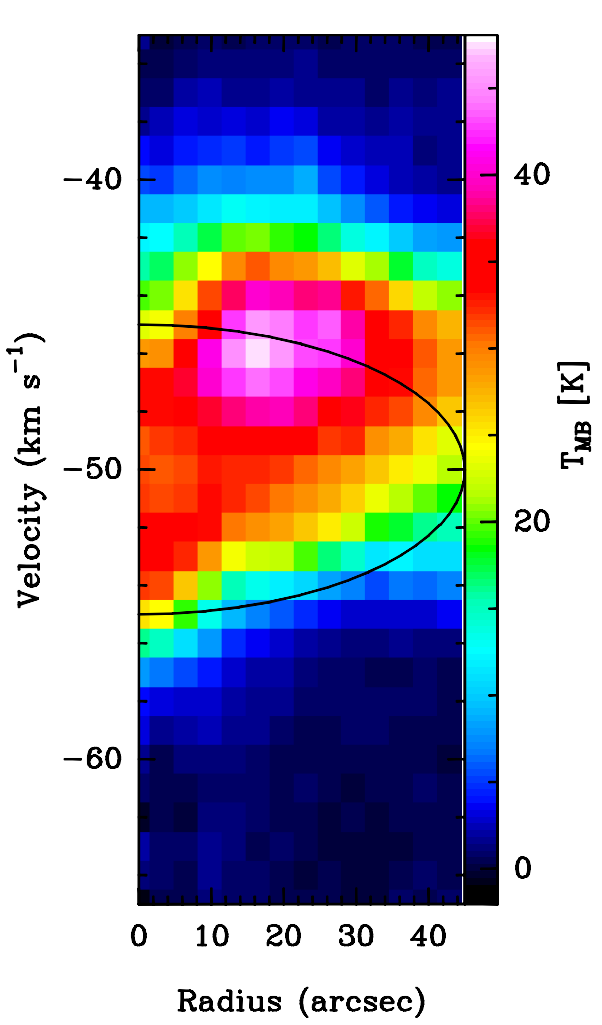

Fig. 10. $(r-v)$ diagram of the HII region G327.3-0.5 obtained from the ${ }^{12} \mathrm{CO}(6-5)$ data cube. The radius axis is the distance to the shell expansion centre, chosen to be the peak of the $\mathrm{cm}$ continuum emission. The half ellipse represents an ideal shell in $(r-v)$ diagram with an expansion velocity of $5 \mathrm{~km} \mathrm{~s}^{-1}$.

Assuming that the ${ }^{12} \mathrm{CO}(6-5)$ emission is optically thick and that the ${ }^{12} \mathrm{CO}(6-5)$ and ${ }^{13} \mathrm{CO}(6-5)$ lines have the same excitation temperatures, the optical depth of the ${ }^{13} \mathrm{CO}(6-5)$ transition, $\tau_{13} \mathrm{CO}$, is

$\tau^{13} \mathrm{CO}=-\ln \left(1-\frac{T_{L}\left({ }^{13} \mathrm{CO}\right)}{T_{L}\left({ }^{12} \mathrm{CO}\right)}\right)$.

The optical depth of the ${ }^{12} \mathrm{CO}(6-5)$ transition can then be obtained by multiplying for the abundance of ${ }^{12} \mathrm{CO}$ relative to ${ }^{13} \mathrm{CO}, X^{12} \mathrm{CO} /{ }^{13} \mathrm{CO} \sim 60$ (Wilson \& Rood 1994). From the optical depth of the ${ }^{12} \mathrm{CO}(6-5)$ line, one can also derive its excitation temperature using Eq. (1). Figure 11 shows the distribution of the optical depth of the ${ }^{13} \mathrm{CO}(6-5)$ line and of the excitation temperature of ${ }^{12} \mathrm{CO}(6-5)$. The ${ }^{13} \mathrm{CO}(6-5)$ emission is moderately optically thick (0.6-0.7) at the HII region and at the infrared dark cloud, while it reaches values of $\sim 1.2$ at the hot core position and in a small part of ring around the HII region. The map distribution of the excitation temperature of the ${ }^{12} \mathrm{CO}(6-5)$ line is shown in the bottom panel of Fig. 11. The map is dominated by the HII region, where $T_{\mathrm{ex}}$ reaches values of $80 \mathrm{~K}$ in the ring around the HII region and then decreases with increasing distance from it. The hot core and the rest of the infrared dark cloud have values around $30-35 \mathrm{~K}$. The excitation temperature increases to the south west of the hot core, in a region where there is also $8 \mu \mathrm{m}$ emission, and to the north-east of the HII along a layer of gas also visible in the ${ }^{12} \mathrm{CO}(6-5)$ integrated intensity map (see Fig. 2), but more prominent in the $T_{\text {ex }}$ map and in the $8 \mu \mathrm{m}$ emission map (see Fig. 1).

From the optical depth and the excitation temperature of the ${ }^{12} \mathrm{CO}(6-5)$ line, we derived the $\mathrm{H}_{2}$ column density assuming a 


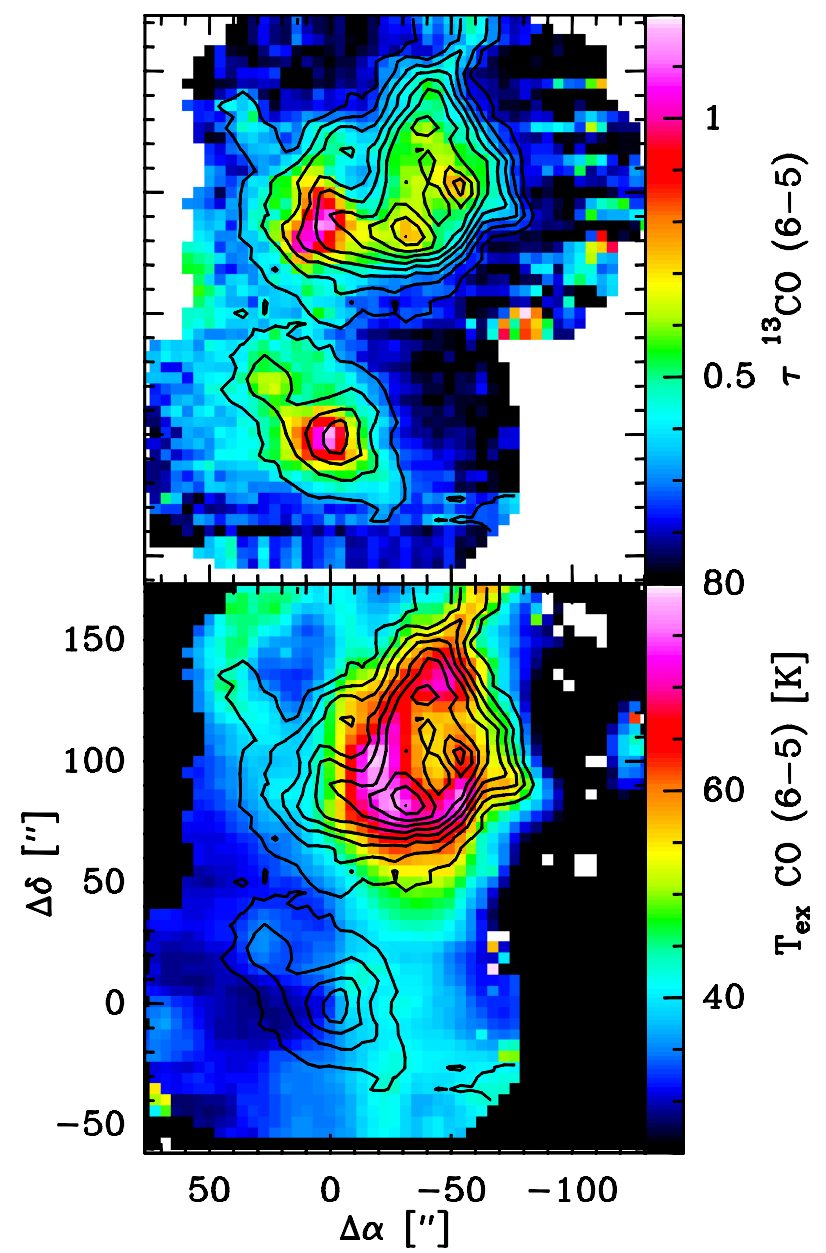

Fig. 11. Distribution of the optical depth of the ${ }^{13} \mathrm{CO}(6-5)$ line $\left(\tau_{13} \mathrm{CO}\right.$, top panel) and of the excitation temperature of ${ }^{12} \mathrm{CO}(6-5)$ transition ( $T_{\mathrm{exCO}}$, bottom panel). Black contours are the ${ }^{13} \mathrm{CO}(6-5)$ integrated intensity as in Fig. 3.

relative abundance of ${ }^{12} \mathrm{CO}$ relative to $\mathrm{H}_{2}$ of $2.7 \times 10^{-4}$ (Lacy et al. 1994). Results are shown in Fig. 12. The largest column density is found towards the hot core $\left(\sim 3 \times 10^{22} \mathrm{~cm}^{-2}\right.$ in the 9.' 4 beam of the ${ }^{13} \mathrm{CO}(6-5)$ data) and decreases along the infrared dark cloud with a distribution similar to that of the $870 \mu \mathrm{m}$ continuum emission. Three peaks around $10^{22} \mathrm{~cm}^{-2}$ are found in the HII region.

We cross-checked our results by computing the $\mathrm{H}_{2}$ column density under the assumption that the ${ }^{13} \mathrm{CO}$ emission is optically thin. The results are consistent with those presented in Fig. 12; the largest differences (of the order of 30\%) are found towards those positions where the optical depth of the ${ }^{13} \mathrm{CO}(6-5)$ transition (Fig. 11) exceeds $\sim 0.7$. The assumption of optically thin emission for ${ }^{13} \mathrm{CO}$ may be particularly useful for the inter-clump medium (arbitrarily defined as the region in the map where the ${ }^{13} \mathrm{CO}$ lines are not detected on individual spectra), towards which we infer $\mathrm{H}_{2}$ column densities of $\sim 2 \times 10^{21} \mathrm{~cm}^{-2}$ corresponding to a ${ }^{13} \mathrm{CO}$ column density of $\sim 10^{16} \mathrm{~cm}^{-2}$ (see also Sect. 5.1).

We also computed column densities and rotational temperatures in the region using the rotational diagram technique applied to the ${ }^{13} \mathrm{CO}$ data. We did not include the ${ }^{12} \mathrm{CO}$ lines in the analysis because of their complex line profiles and high optical depths. Given the optical depth previously derived for the ${ }^{13} \mathrm{CO}(6-5)$ line, we did not apply any correction due to optical

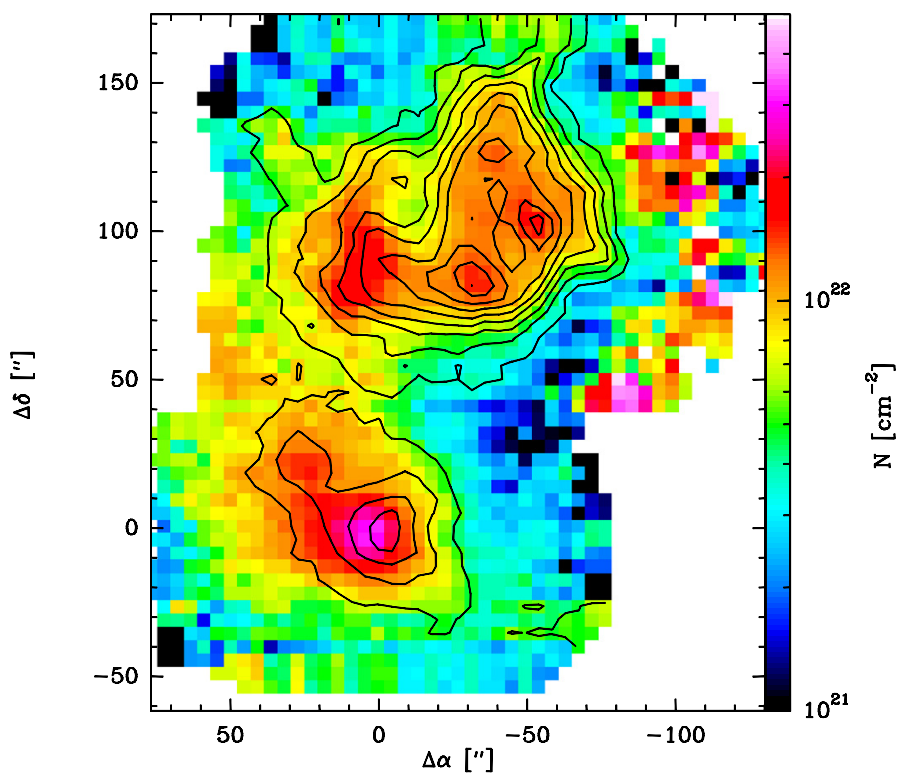

Fig. 12. Distribution of the $\mathrm{H}_{2}$ column density in the G327.3-0.6 starforming region based on equation 1 . Black contours are the ${ }^{13} \mathrm{CO}(6-5)$ integrated intensity as in Fig. 3.

depth effects to the ${ }^{13} \mathrm{CO}$ data. Results are consistent with the estimates based on Eq. (1). The main differences between the two analyses are found towards the hot core, where the rotational temperature is higher than the excitation derived with Eq. (1) ( $T_{\text {rot }} \sim 70 \mathrm{~K}$ and $T_{\mathrm{ex}} \sim 32 \mathrm{~K}$ ) and the $\mathrm{H}_{2}$ column density lower $\left(10^{22} \mathrm{~cm}^{-2}\right.$ versus $3 \times 10^{22} \mathrm{~cm}^{-2}$ obtained with the first method). The differences between the two methods are likely influenced by the self-absorption profile detected in the ${ }^{12} \mathrm{CO}(6-5)$ line which results in lower line intensities towards the regions of large column densities. In particular, while the first approach overestimates the optical depths (and hence column density) because of the self absorption, the column density derived with the rotational diagram analysis is likely underestimated towards the hot core because of the optically thin emission assumption, and should be corrected by a factor $\tau_{\text {LTE }} /\left(1-\exp \left(\tau_{\text {LTE }}\right)-\right) \sim 1.7$.

Finally, we computed the ratio between the amount of warm gas (traced by ${ }^{12} \mathrm{CO}$ and ${ }^{13} \mathrm{CO}(6-5)$ and shown in Fig. 12) and the total amount of gas (traced by the continuum emission at $870 \mu \mathrm{m})$ as derived assuming a dust temperature equal to the excitation temperature of ${ }^{12} \mathrm{CO}(6-5)$ and a dust opacity of $0.0182 \mathrm{~cm}^{2} \mathrm{~g}^{1}$ (Kauffmann et al. 2008). The results are shown in Fig. 13: the warm gas is only a small percentage $(\sim 10 \%)$ of the total gas in the infrared dark cloud, while it reaches values up to $\sim 35 \%$ of the total gas in the ring surrounding the HII region.

\section{2. ${ }^{13} \mathrm{CO}$ ladder}

Since the G327.3-0.6 region was mapped in three different transitions of the ${ }^{13} \mathrm{CO}$ molecule, we can perform a multi-line analysis towards selected positions and infer the parameter of the gas. The advantage of ${ }^{13} \mathrm{CO}$ compared to the main isotopologue is in the lower opacities of the lines and in the less complex line profiles. For this analysis, we used the RADEX program (van der Tak et al. 2007) with expanding sphere geometry. The molecular dataset comes from the LAMDA database (Schöier et al. 2005) and includes collisional rates adapted from Yang et al. (2010). We ran models with temperatures from 20 to $200 \mathrm{~K}$, densities in the range $10^{4}-5 \times 10^{7} \mathrm{~cm}^{-3}$, and ${ }^{13} \mathrm{CO}$ column densities between $10^{14}$ and $10^{19} \mathrm{~cm}^{-2}$. 


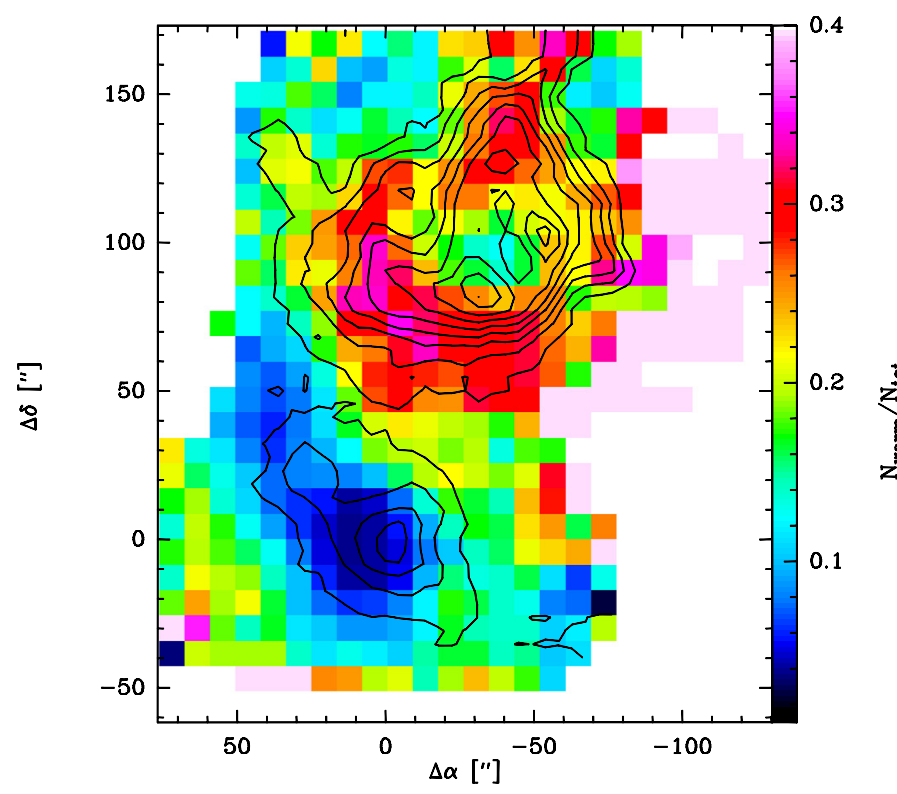

Fig. 13. Distribution of the ratio between the column density of warm gas (traced by ${ }^{12} \mathrm{CO}$ and ${ }^{13} \mathrm{CO}(6-5)$ ) and the total $\mathrm{H}_{2}$ column density (traced by the continuum emission at $870 \mu \mathrm{m}$ ) in the G327.3-0.6 starforming region. Black contours are the ${ }^{13} \mathrm{CO}(6-5)$ integrated intensity as in Fig. 3.

Table 3. Line parameters of the ${ }^{13} \mathrm{CO}$ lines.

\begin{tabular}{lccc}
\hline \hline Position & $\begin{array}{c}v_{\mathrm{LSR}} \\
\left(\mathrm{km} \mathrm{s}^{-1}\right)\end{array}$ & $\begin{array}{c}F W H M \\
\left(\mathrm{~km} \mathrm{~s}^{-1}\right)\end{array}$ & $\begin{array}{c}\int T_{\mathrm{mb}} \delta \mathrm{v} \\
\left(\mathrm{K} \mathrm{km} \mathrm{s}^{-1}\right)\end{array}$ \\
\hline hot core & $-44.74 \pm 0.03$ & $7.23 \pm 0.06$ & $107.8 \pm 0.8$ \\
IRDC & $-45.99 \pm 0.02$ & $5.44 \pm 0.05$ & $67.3 \pm 0.8$ \\
HII & $-48.97 \pm 0.01$ & $8.40 \pm 0.02$ & $202.2 \pm 0.5$ \\
& & ${ }^{13} \mathrm{CO}(8-7)$ \\
hot core & $-44.63 \pm 0.06$ & $6.5 \pm 0.2$ & $43.5 \pm 0.9$ \\
IRDC & $-46.5 \pm 0.2$ & $4.4 \pm 0.6$ & $16 \pm 2$ \\
HII & $-48.83 \pm 0.05$ & $8.2 \pm 0.1$ & $116 \pm 1$ \\
& & ${ }^{13} \mathrm{CO}(10-9)$ & \\
hot core & $-44.28 \pm 0.07$ & $6.4 \pm 0.2$ & $24.4 \pm 0.5$ \\
IRDC & $-46.8 \pm 0.1$ & $3.7 \pm 0.3$ & $4.7 \pm 0.3$ \\
HII & $-49.56 \pm 0.04$ & $7.67 \pm 0.09$ & $52.4 \pm 0.5$ \\
\hline
\end{tabular}

All data were smoothed to the resolution of the ${ }^{13} \mathrm{CO}(10-9)$ map. We selected three positions for the analysis: the hot core, the IRDC position $\left(\left(30^{\prime \prime}, 30^{\prime \prime}\right)\right.$ from the centre of the APEX maps), and the centre of the HII region. The IRDC position was selected to be a position associated with high column density in the infrared dark cloud (see Figs. 2, 3 and 12) but without IR emission. However, it is only $10^{\prime \prime}$ to the north of the EGO candidate (Cyganowski et al. 2008, see Figs. 2, 3), and therefore, given the beam of the observations, contamination from the embedded YSO may still be possible. Table 3 reports the measured line parameters of the ${ }^{13} \mathrm{CO}$ transitions obtained with Gaussian fits; based on these values, we adopt line widths of 6,3 and 7 , for the hot core, the IRDC and the HII respectively, in agreement with values reported by San José-García et al. (2012) for the ${ }^{13} \mathrm{CO}(10-9)$ line towards a sample of intermediate- and high-mass sources. The spectra are shown in Fig. 7.

The results of the RADEX analysis are listed in Table 4 and shown in Fig. 14. For the IRDC position, the ${ }^{13} \mathrm{CO}(10-9)$ line intensity is not well fitted by our one-temperature model. This

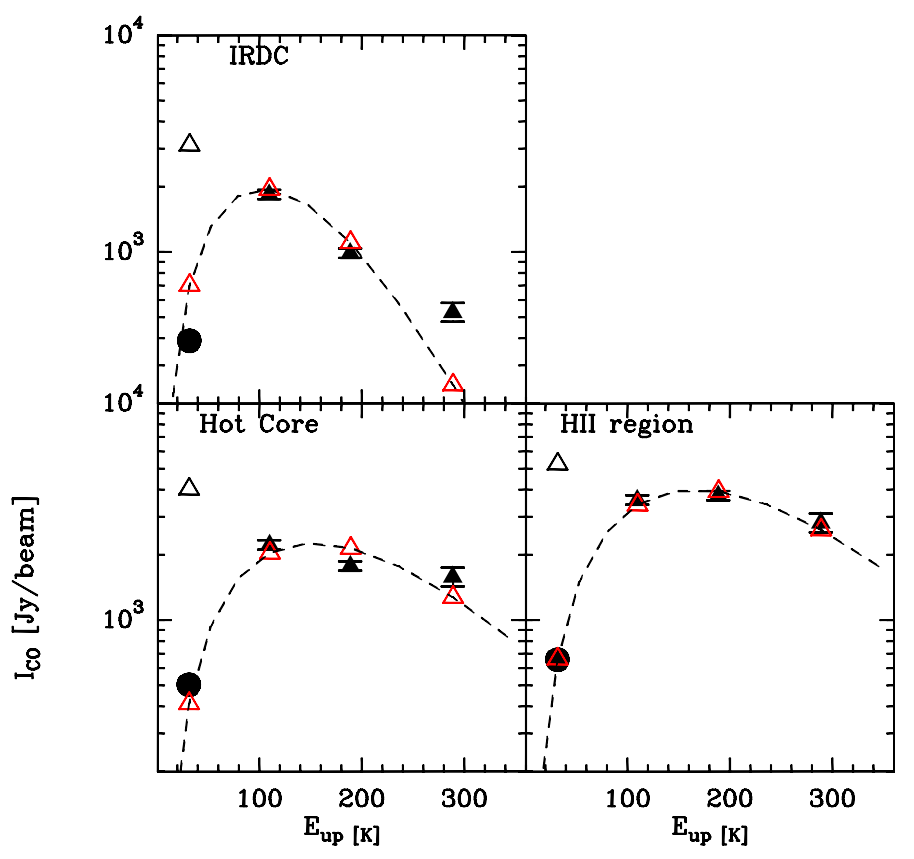

Fig. 14. Distribution of the ${ }^{13} \mathrm{CO}$ peak line intensities. Full black triangles correspond to ${ }^{13} \mathrm{CO}(6-5),(8-7)$ and (10-9) observed intensities. The circle represents the observed $\mathrm{C}^{18} \mathrm{O}(3-2)$ flux, the empty black triangle the flux of the $\mathrm{C}^{18} \mathrm{O}(3-2)$ line multiplied by $X_{13} \mathrm{CO} / \mathrm{C}^{18} \mathrm{O} \sim 8$. The red triangles are the best model fit results. The error bars include only calibration uncertainties. The dashed lines represent the best fit ${ }^{13} \mathrm{CO}$ ladder.

Table 4. Best fit parameters of the ${ }^{13} \mathrm{CO}$ line modelling.

\begin{tabular}{lccc}
\hline \hline Position & $\begin{array}{c}T \\
(\mathrm{~K})\end{array}$ & $\begin{array}{c}n_{\mathrm{H}_{2}} \\
\left(\mathrm{~cm}^{-3}\right)\end{array}$ & $\begin{array}{c}N_{{ }_{13} \mathrm{CO}} \\
\left(\mathrm{cm}^{-2}\right)\end{array}$ \\
\hline Hot core & $70(>60)$ & $1 \times 10^{7}\left(>10^{4}\right)$ & $5 \times 10^{16}$ \\
IRDC & $45_{-11}^{+20}$ & $1 \times 10^{6}\left(>10^{5}\right)$ & $5 \times 10^{16}$ \\
HII & $75_{-7}^{+55}$ & $5 \times 10^{6}\left(>4 \times 10^{4}\right)$ & $1 \times 10^{17}$ \\
\hline
\end{tabular}

Notes. The errors represent the $3 \sigma$ confidence levels in the temperaturedensity plane or $3 \sigma$ lower (shown in brackets) limit when no stronger constraints can be inferred.

likely reflects the fact that the ${ }^{13} \mathrm{CO}(10-9)$ spectrum is dominated by the embedded source while the other two lines sample colder gas in the envelope. We considered a $20 \%$ calibration error for the ${ }^{13} \mathrm{CO}(6-5)$ and $(8-7)$ observations and a $15 \%$ error for the ${ }^{13} \mathrm{CO}(10-9)$ data. Wyrowski et al. (2006) mapped the region with the APEX telescope in the $\mathrm{C}^{18} \mathrm{O}(3-2)$ line. Therefore, since no observations were performed in the ${ }^{13} \mathrm{CO}(3-2)$ transition, we included the $\mathrm{C}^{18} \mathrm{O}(3-2)$ data in Fig. 14. Note however, that the $\mathrm{C}^{18} \mathrm{O}(3-2)$ fluxes are not included in the fitting procedure, but that they are simply used to cross-check results. Since the $\mathrm{C}^{18} \mathrm{O}(3-2)$ flux corresponds to a lower limit to the flux of ${ }^{13} \mathrm{CO}(3-2)$ line, we also plotted the $\mathrm{C}^{18} \mathrm{O}(3-2)$ flux corrected for the abundance ratio of ${ }^{13} \mathrm{CO}$ to $\mathrm{C}^{18} \mathrm{O}, X_{13} \mathrm{CO} / \mathrm{C}^{18} \mathrm{O} \sim 8$ (Wilson $\&$ Rood 1994). This value is likely an upper limit to the flux of the ${ }^{13} \mathrm{CO}(3-2)$ line due to opacity effects.

An example of the $\chi^{2}$ distribution projected on to the $T-n$ plane is shown in Fig. 15 for the HII position, where the reduced $\chi^{2}$ at the best fit position is 3 . Figure 15 shows the typical inverse $n-T$ relationship often seen in $\chi^{2}$ distributions and 
S. Leurini et al.: Warm gas in the G327.3-0.6 massive star-forming region

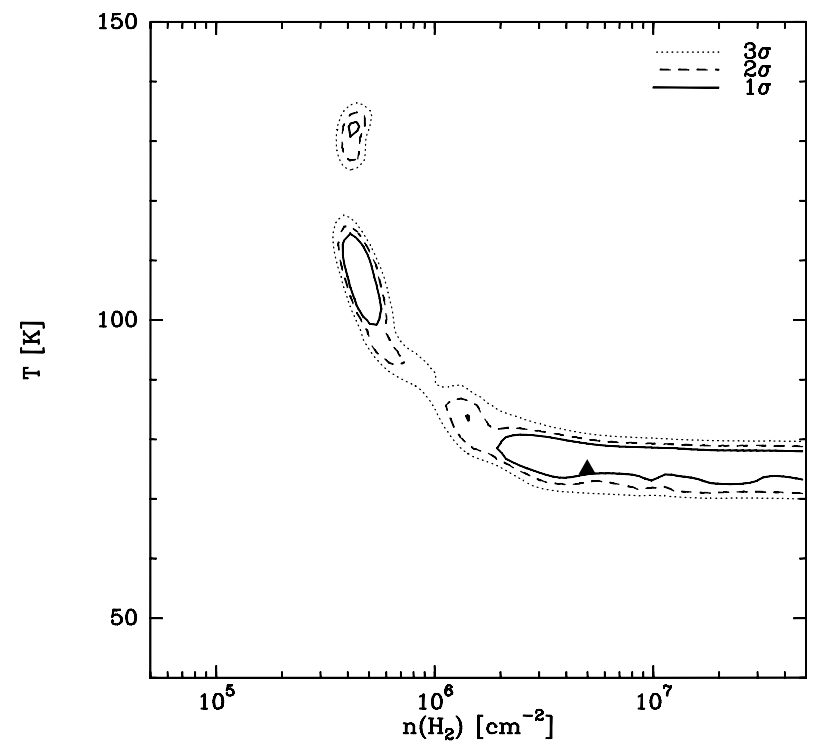

Fig. 15. Projection of the 3-dimensional (T-n-N) distribution of the $\chi^{2}$ on the $T-n$ plane for the HII position. The contours show the 1,2 and $3 \sigma$ confidence levels for two degrees of freedom. The triangle marks the best fit position.

due to the fact that density and temperature are, in the case of the $\mathrm{CO}$ molecule, not independent parameters (see Appendix $\mathrm{C}$ of van der Tak et al. 2007).

We note here that the detection of the ${ }^{13} \mathrm{CO}(10-9)$ line breaks the degeneracy between density and temperature typical of ${ }^{12} \mathrm{CO}$ analyses (e.g., Kramer et al. 2004; van der Tak et al. 2007) and help to give stronger constraints: indeed, with the exception of the hot core, at least the temperature of the gas is well determined at all positions.

The results of the RADEX analysis confirm that the LTE assumption used in Sect. 4.1 is reasonable since the inferred densities are much larger than the critical densities (a few $10^{4} \mathrm{~cm}^{-3}$ for all three analysed transitions). Assuming $X^{12} \mathrm{CO} /{ }^{13} \mathrm{CO} \sim 60$ and an abundance of ${ }^{12} \mathrm{CO}$ relative to $\mathrm{H}_{2}$ of $2.7 \times 10^{-4}$, the derived ${ }^{13} \mathrm{CO}$ column densities listed in Table 4 correspond to $\mathrm{H}_{2}$ column densities of some $10^{22} \mathrm{~cm}^{-2}$ for the hot core and the IRDC position, and of $2 \times 10^{22} \mathrm{~cm}^{-2}$ for the HII position. The derived column densities and temperatures are in agreement with those derived in Sect. 4.1 through rotational diagrams of the ${ }^{13} \mathrm{CO}$ emission. The ${ }^{13} \mathrm{CO}(6-5)$ optical depths are also in agreement with those estimated in Sect. 4.1 through the ${ }^{13} \mathrm{CO}$ and ${ }^{12} \mathrm{CO}(6-5)$ line ratio, with the exception of the hot core position: $\tau_{\text {LTE }} \sim 1.2$ and $\tau_{\text {RADEX }} \sim 0.3$ for the hot core, 0.6 and 0.5 for the HII region, and 0.6 and 0.7 for the IRDC position.

We finally stress that the results obtained with the LTE analysis (Sect. 4.1) and with the RADEX code (this section) are based on the assumptions that 1) all lines have a beam filling factor of one, and 2) that the emitting gas is homogeneous, whereas selfabsorption profiles in the ${ }^{12} \mathrm{CO}$ lines indicate an excitation gradient along the line of sights. For optically thin lines $\left({ }^{13} \mathrm{CO}\right.$ in the current case), a beam filling factor less than one (but equal for all transitions) would mostly affect the column density and result in larger values of $N$; for optically thick lines, a smaller value of $\eta$ would imply larger values of density and/or temperature.

The uncertainties on the derived parameters due to the assumption of a homogeneous medium are of less immediate interpretation, and more complex radiative transfer codes (e.g., Hogerheijde \& van der Tak 2000) should be used to reproduce the observed line velocity profiles.
Table 5. Percentage of integrated fluxes from the HII region G327.3-0.5 and from the inter-clump gas respect to the total integrated flux in the map.

\begin{tabular}{lcc}
\hline \hline Line & HII & Inter \\
\hline $\mathrm{CO}(3-2)$ & $56 \%$ & $10 \%$ \\
$\mathrm{CO}(6-5)$ & $58 \%$ & $10 \%$ \\
$\mathrm{CO}(7-6)$ & $60 \%$ & $10 \%$ \\
${ }^{13} \mathrm{CO}(6-5)$ & $49 \%$ & $7 \%$ \\
${ }^{13} \mathrm{CO}(8-7)$ & $67 \%$ & $5 \%$ \\
${ }^{13} \mathrm{CO}(10-9)$ & $85 \%$ & $2 \%$ \\
\hline
\end{tabular}

\section{Discussion}

\subsection{Total ${ }^{12} \mathrm{CO}$ and ${ }^{13} \mathrm{CO}$ emission}

Figure 16 shows the ${ }^{12} \mathrm{CO}$ and ${ }^{13} \mathrm{CO}$ ladders obtained by averaging the emission of the different observed transitions, smoothed to the resolution of the HIFI data, over four regions: the total map, the HII region G327.3-0.5, the IRDC hosting the hot core (the selected region does include the hot core), and finally the inter-clump gas, which was defined as the region in the map where the ${ }^{13} \mathrm{CO}$ lines are not detected on individual spectra. This region has an equivalent radius of $50^{\prime \prime}$ (corresponding to $\sim 0.8 \mathrm{pc}$ at the distance of the source). Examples of ${ }^{12} \mathrm{CO}$ line profiles towards the inter-clump gas are shown in Fig. 17. The $\mathrm{C}^{18} \mathrm{O}(3-2)$ cannot be used in this analysis because the observations cover a much smaller region than that mapped in ${ }^{13} \mathrm{CO}$. The total mass of the mapped region can be computed using the excitation temperature and $\mathrm{H}_{2}$ column density distributions shown in Figs. 11 and 12 . This corresponds to $\sim 700 M_{\odot}$.

The four regions have similar ${ }^{12} \mathrm{CO}$ and ${ }^{13} \mathrm{CO}$ ladders. In order to correct for self-absorption, integrated fluxes were obtained for each of the four regions from line fitting of the $\mathrm{CO}$ spectra with one Gaussian component. While this works fine for the spectra of the IRDC hosting the hot core and for the interclump gas, the line profiles of the total map and of HII region are red-skewed and therefore the fluxes derived with this method are likely underestimated. For the main isotopologue, the flux of the (7-6) and (6-5) transitions is very similar, although for the IRDC + HC and the inter-clump the flux of the (7-6) line is lower than that of the (6-5) line. On the other hand, in the ${ }^{13} \mathrm{CO}$ ladder the peak flux decreases with increasing energy level. For all transitions presented in this paper, the spectra are dominated in intensity by the HII region, whose flux is of the order of $60 \%$ of the total flux for the main isotopologue lines, and ranging from $\sim 49 \%$ of the total flux in the $(6-5)$ line to $\sim 85 \%$ in the (10-9) transition for ${ }^{13} \mathrm{CO}$ (see Table 5). The main difference between the ${ }^{12} \mathrm{CO}$ spectra and those of ${ }^{13} \mathrm{CO}$ lies in the emission from the inter-clump gas: for all ${ }^{12} \mathrm{CO}$ lines, the intensity is relatively strong, $10 \%$ of the flux from the whole region. On the other hand, the flux of the ${ }^{13} \mathrm{CO}$ lines coming from the inter-clump gas decreases with increasing $J$, from $\sim 7 \%$ of the total flux for $J=6$ to $\sim 2 \%$ for $J=10$. The general behaviour of the ${ }^{12} \mathrm{CO}$ and ${ }^{13} \mathrm{CO}$ ladders is qualitatively compatible with PDR models from Koester et al. (1994) for high density $\left(10^{6}-10^{7} \mathrm{~cm}^{-3}\right)$ clouds illuminated on one side by a UV radiation field (their model B). In Fig. 16, we show the predicted CO and ${ }^{13} \mathrm{CO}$ line intensities for models with a density of $10^{7} \mathrm{~cm}^{-3}$, incident UV fields with strength $10^{3}$ and $10^{4}$ relative to the average interstellar field (Draine 1978), a visual extinction of 10, and a Doppler broadening of 3 and $1 \mathrm{~km} \mathrm{~s}^{-1}$. High densities ( $n>10^{6} \mathrm{~cm}^{-3}$, in agreement with our results from Sect. 4.2) are 

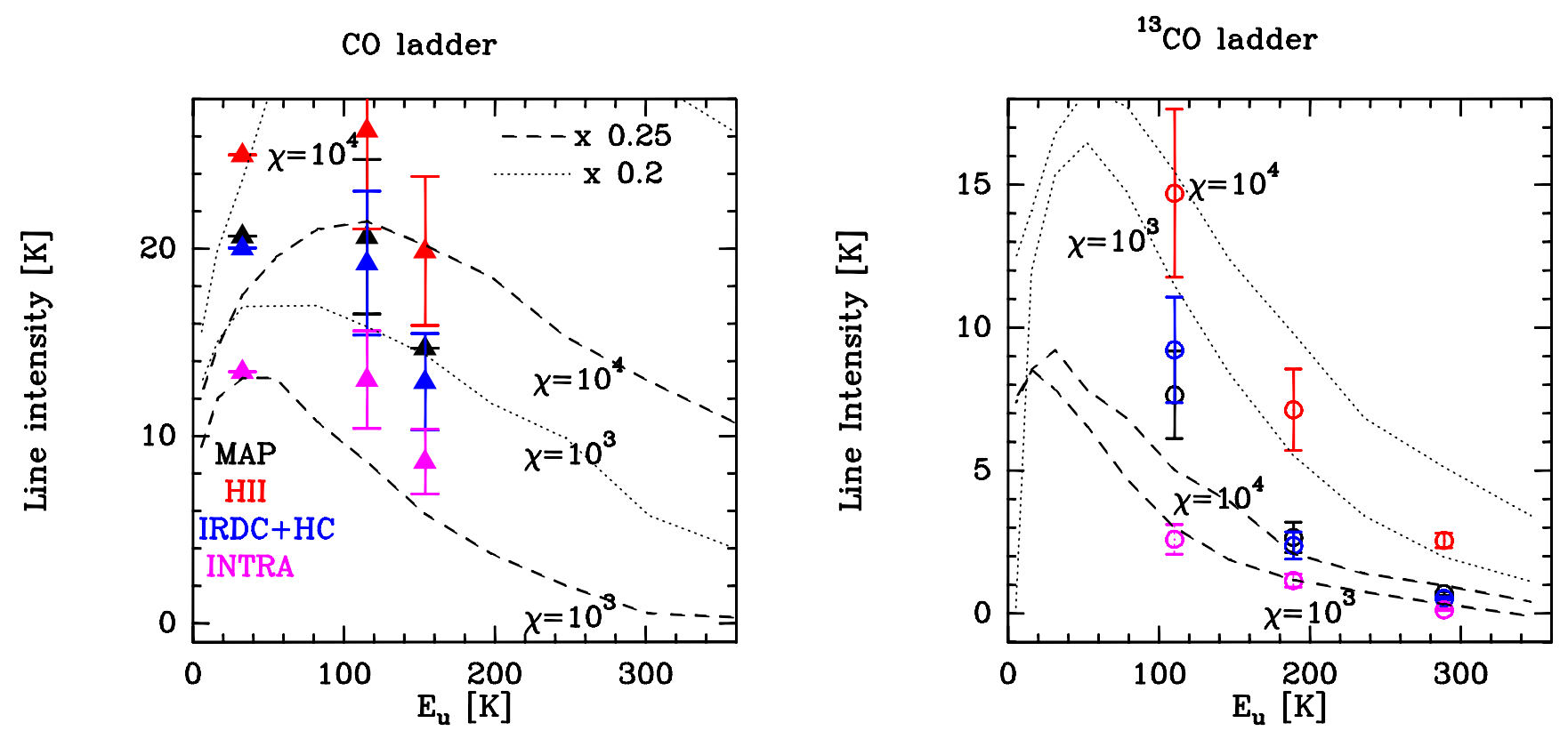

Fig. 16. $\mathrm{CO}$ (left) and ${ }^{13} \mathrm{CO}$ (right) ladders for the whole mapped region (black), the HII region G327.3-0.5 (red), the IRDC including the hot core (blue) and the inter-clump gas (magenta). Error bars include only calibration uncertainties. In both panels, the dashed and dotted curves represent the predicted intensities for model B from Koester et al. (1994) for a density of $10^{7} \mathrm{~cm}^{-3}$, incident UV fields of $10^{3}$ and $10^{4}$ relative to the average interstellar field, a visual extinction of 10, and a Doppler broadening of 3 (dashed curve) and $1 \mathrm{~km} \mathrm{~s}^{-1}$ (dotted curve).

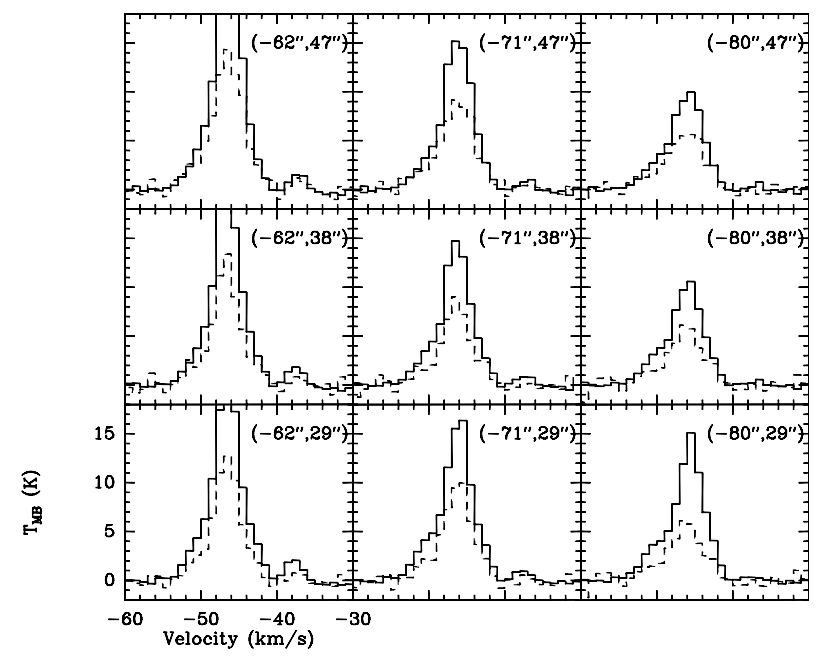

Fig. 17. $\mathrm{CO}(6-5)$ (solid line) and ${ }^{12} \mathrm{CO}(7-6)$ (dashed line) spectra towards some positions in the inter-clump gas. The offset position from the centre of the APEX ${ }^{12} \mathrm{CO}$ maps (Sect. 2.1) is shown for each spectrum in the top right corner.

needed to locate the peak of the CO ladders at mid-, high- $J$ transitions (see Figs. 9-10, 12-13 of Koester et al. 1994). Stronger UV radiation fields also shift the peak of the $\mathrm{CO}$ ladders to higher $J$ transitions than observed. In Fig. 16, the ${ }^{12} \mathrm{CO}$ model intensities are corrected by a factor 0.2 and 0.25 to correct for different line-widths between the model and the observations, and possibly for beam dilution effects. On the other hand, the ${ }^{13} \mathrm{CO}$ results of Fig. 16 are not scaled down by any factor as they are far too weak to match the observations. Koester et al. (1994) already noticed that the predicted line intensities of mid- $J$ ${ }^{13} \mathrm{CO}$ transitions in their models are much weaker than observed in star-forming regions. They proposed that mid- $J{ }^{13} \mathrm{CO}$ emission comes from a large number of filamentary structures, or clumps, along the line of sight. In this way, the modelled line intensity of mid- $J{ }^{13} \mathrm{CO}$ lines would increase significantly as the lines are optically thin, while it would not change for optically thick transitions.

Rotational diagrams of the ${ }^{13} \mathrm{CO}$ emission applied to the spectra of the HII region, of the IRDC and of the inter-clump gas infer rotational temperatures of $66 \mathrm{~K}, 47 \mathrm{~K}$ and $44 \mathrm{~K}$, respectively, and ${ }^{13} \mathrm{CO}$ column densities of $6 \times 10^{15} \mathrm{~cm}^{-2}$ for the HII region and the IRDC, and of $2 \times 10^{15} \mathrm{~cm}^{-2}$ for the interclump gas. Since the inter-clump gas has physical parameters very similar to those of the IRDC region, but a much lower column density, we suggest that it is composed of high-density clumps with low filling factors. This is again in agreement with PDR models (e.g., Koester et al. 1994; Cubick et al. 2008) which predict strong emission at mid- $J{ }^{13} \mathrm{CO}$ and high- $J{ }^{12} \mathrm{CO}$ lines in the case of small, low mass, high density clumps.

Cubick et al. (2008) suggested that the COBE ${ }^{12} \mathrm{CO}$ ladder of the Milky Way can be reproduced by a clumpy PDR model, and that the bulk of the Galactic FIR line emission comes from PDRs around the Galactic population of massive stars. Our observations seem to confirm this result, since the $\mathrm{CO}$ emission of the G327.3-0.6 region is dominated by the PDR around the HII region. Our results are also consistent with the findings from Davies et al. (2011) and Mottram et al. (2011). These authors studied the properties of massive YSOs and compact HII regions in the RMS survey (Hoare et al. 2005), and found that there is no significant population of massive YSOs above $\sim 10^{5} L_{\odot}$, while compact HII regions are detected up to $\sim 10^{6} L_{\odot}$. Since high- $J$ CO lines are among the most important cooling lines in PDR, they reflect the luminosity of their heating sources: if the luminosity distribution of massive stars in the Galaxy is dominated by HII regions and not by younger massive stars, then we also expect that the $\mathrm{CO}$ distribution follows the same rule.

\subsection{Comparisons with other star forming regions}

Large-scale mapping of some low- and high-mass star forming regions was performed in several ${ }^{12} \mathrm{CO}$ transitions. However, 
given the different critical densities of the lines, we prefer to compare our results with studies carried on with $J>3 \mathrm{CO}$ lines. Excitation temperatures around $8-30 \mathrm{~K}$ are found in extended diffuse emission and towards the brightest positions, respectively, in low-mass star forming regions (e.g., Davis et al. 2010; Curtis et al. 2010), while massive star-forming regions are usually warmer as confirmed by our findings (see also Wilson et al. 2001; Jakob et al. 2007; Kirk et al. 2010; Wilson et al. 2011; Peng et al. 2012). The CO column densities $\left(10^{17}-10^{18} \mathrm{~cm}^{-2}\right.$ from the LTE analysis) found in G327 are also comparable with values obtained in other massive star-forming regions (Wilson et al. 2001; Jakob et al. 2007; White et al. 2010; Wilson et al. 2011).

Comparison with ${ }^{12} \mathrm{CO}$ large maps of other high-mass star forming regions would be important to verify whether our result that the ${ }^{12} \mathrm{CO}$ distribution is dominated by the HII region is a common feature or not. However, most studies do not cover different evolutionary phases as in our case. For OMC-1, Peng et al. (2012) confirmed that the peak of the integrated intensity of several CO isotopologue lines is close to the Orion-KL hot core (although Orion-south and the Orion Bar PDR are also very prominent). One should notice however, that Orion is roughly six times closer to the Sun than G327.3-0.6, and therefore the Orion Bar and Orion-KL would be much closer on sky $\left(\sim 30^{\prime \prime}\right)$ if one would place them at the distance of G327.3-0.6. Moreover, Orion-KL likely represents a special case since it hosts a very powerful outflow (e.g., Kwan \& Scoville 1976; Snell et al. 1984), which could alter the distribution of ${ }^{12} \mathrm{CO}$ in the region. Indeed, Marrone et al. (2004) show that broad velocity emission arises mainly from the Orion-KL region, while much of the narrower emission arises from the PDR excited by the M42 HII region.

\subsection{Self-absorption profiles}

As noted in Sect. 3.2, all ${ }^{12} \mathrm{CO}$ transitions analysed in this paper are affected by self-absorption, which is likely to be due to cold gas surrounding a warmer component (Phillips et al. 1981). In particular along the infrared dark cloud (Fig. 8), the ${ }^{12} \mathrm{CO}(6-5)$ line has blue-skewed profiles in the north-east (towards the EGO and the IRDC positions) and the red-skewed ones towards the south-west (the hot core). Blue- and red-skewed profiles (e.g., Mardones et al. 1997) are commonly interpreted as due to rotation or outflow motions (which should produce equal numbers of red and blue profiles, and could therefore explain the profiles detected towards the EGO and IRDC position, the hot core and the HII region, Fig. 6) or to infall (which should produce profiles which are skewed towards the blue, e.g. towards the EGO and IRDC position) or to expansion (which should produce profiles skewed towards the red and could be responsible for the ${ }^{12} \mathrm{CO}$ spectra of the hot core and the HII region). From the PV diagram of the ${ }^{12} \mathrm{CO}(6-5)$ line, we do not have any evidence of global rotation towards the hot core and the red-skewed profile can be interpreted in terms of expansion or outflow motion. Similarly, from Fig. 10 we see that the emission does not follow a perfect expanding spherical shell which might imply that the rotation is on the origin of the self-absorbed profile seen towards the HII region. Finally, the blue-skewed line profile detected toward the EGO and IRDC positions are typical of infall motion.

\section{Conclusions}

To study the effect of feedback from massive star forming regions in their surrounding environment, we selected the region G327.3-0.6 for large scale mapping of several mid- $J{ }^{12} \mathrm{CO}$ and ${ }^{13} \mathrm{CO}$ lines with the APEX telescope and of the high- $J$ ${ }^{13} \mathrm{CO}(10-9)$ transition with the Herschel satellite. Our results can be summarised as follows:

1. maps of all transitions are dominated by the PDR associated with the HII region G327.3-0.5; mid- $J{ }^{12} \mathrm{CO}$ and ${ }^{13} \mathrm{CO}$ emission is detected along the whole extent of the IRDC;

2 . mid- $J$ transitions show rather extended emission with typical excitation temperatures of $\sim 30 \mathrm{~K}$ and column densities of some $10^{21} \mathrm{~cm}^{-2}$;

3. all observed transitions are detected also in the inter-clump gas when averaged over large regions. The inter-clump emission is compatible with LTE emission from a gas at $44 \mathrm{~K}$, and with a ${ }^{13} \mathrm{CO}$ column density of $2 \times 10^{15} \mathrm{~cm}^{-2}$, thus suggesting that the inter-clump is composed of high-density clumps with low filling factors;

4. the warm gas traced by ${ }^{12}$ and ${ }^{13} \mathrm{CO}(6-5)$ is only a small percentage $(\sim 10 \%)$ of the total gas in the infrared dark cloud, while it reaches values up to $\sim 35 \%$ of the total gas in the ring surrounding the HII region;

5. the ${ }^{12} \mathrm{CO}$ and ${ }^{13} \mathrm{CO}$ ladders are qualitatively compatible with PDR models for high density gas $\left(n>10^{6} \mathrm{~cm}^{-3}\right)$ and, in the case of ${ }^{13} \mathrm{CO}$, suggest that the emission comes from a large number of clumps;

6. the detection of the ${ }^{13} \mathrm{CO}(10-9)$ line allows to give stronger constraints on the physics of the gas by breaking the degeneracy between density and temperature (typical of ${ }^{12} \mathrm{CO}$ and ${ }^{13} \mathrm{CO}$ transitions) in the high temperature-low density part of the $T-n$ plane.

Acknowledgements. The authors thank Dr. Joe Mottram for a careful review of the manuscript and an anonymous referee for useful comments and suggestions. Herschel is an ESA space observatory with science instruments provided by European-led Principal Investigator consortia and with important participation from NASA. HIFI has been designed and built by a consortium of institutes and university departments from across Europe, Canada and the United States under the leadership of SRON Netherlands Institute for Space Research, Groningen, The Netherlands and with major contributions from Germany, France and the US. Consortium members are: Canada: CSA, U.Waterloo; France: CESR, LAB, LERMA, IRAM; Germany: KOSMA, MPIfR, MPS; Ireland, NUI Maynooth; Italy: ASI, IFSI-INAF, Osservatorio Astrofisico di Arcetri- INAF; Netherlands: SRON, TUD; Poland: CAMK, CBK; Spain: Observatorio Astronómico Nacional (IGN), Centro de Astrobiología (CSIC-INTA). Sweden: Chalmers University of Technology - MC2, RSS \& GARD; Onsala Space Observatory; Swedish National Space Board, Stockholm University - Stockholm Observatory; Switzerland: ETH Zurich, FHNW; USA: Caltech, JPL, NHSC.

\section{References}

Beuther, H., Schilke, P., Sridharan, T. K., et al. 2002, A\&A, 383, 892 Blitz, L., \& Stark, A. A. 1986, ApJ, 300, L89

Cubick, M., Stutzki, J., Ossenkopf, V., Kramer, C., \& Röllig, M. 2008, A\&A, 488,623

Curtis, E. I., Richer, J. S., \& Buckle, J. V. 2010, MNRAS, 401, 455

Cyganowski, C. J., Whitney, B. A., Holden, E., et al. 2008, AJ, 136, 2391

Davies, B., Hoare, M. G., Lumsden, S. L., et al. 2011, MNRAS, 1015

Davis, C. J., Chrysostomou, A., Hatchell, J., et al. 2010, MNRAS, 405, 759

de Graauw, T., Helmich, F. P., Phillips, T. G., et al. 2010, A\&A, 518, L6

Draine, B. T. 1978, ApJS, 36, 595

Gibb, E., Nummelin, A., Irvine, W. M., Whittet, D. C. B., \& Bergman, P. 2000, ApJ, 545, 309

Gooch, R. 1996, in Astronomical Data Analysis Software and Systems V, eds. G. H. Jacoby, \& J. Barnes, ASP Conf. Ser., 101, 80

Goss, W. M. \& Shaver, P. A. 1970, Aust. J. Phys. Astrophys. Suppl., 14, 1

Güsten, R., Baryshev, A., Bell, A., et al. 2008, in SPIE Conf. Ser. 7020

Hoare, M. G., Lumsden, S. L., Oudmaijer, R. D., et al. 2005, in Massive Star Birth: A Crossroads of Astrophysics, eds. R. Cesaroni, M. Felli, E. Churchwell, \& M. Walmsley, IAU Symp., 227, 370

Hogerheijde, M. R. \& van der Tak, F. F. S. 2000, A\&A, 362, 697

Jakob, H., Kramer, C., Simon, R., et al. 2007, A\&A, 461, 999 
Kasemann, C., Güsten, R., Heyminck, S., et al. 2006, in SPIE Conf. Ser. 6275 Kauffmann, J., Bertoldi, F., Bourke, T. L., Evans, II, N. J., \& Lee, C. W. 2008, A\&A, 487, 993

Kirk, J. M., Polehampton, E., Anderson, L. D., et al. 2010, A\&A, 518, L82

Klein, B., Philipp, S. D., Krämer, I., et al. 2006, A\&A, 454, L29

Koester, B., Stoerzer, H., Stutzki, J., \& Sternberg, A. 1994, A\&A, 284, 545

Kramer, C., Jakob, H., Mookerjea, B., et al. 2004, A\&A, 424, 887

Kwan, J., \& Scoville, N. 1976, ApJ, 210, L39

Lacy, J. H., Knacke, R., Geballe, T. R., \& Tokunaga, A. T. 1994, ApJ, 428, L69

Mardones, D., Myers, P. C., Tafalla, M., et al. 1997, ApJ, 489, 719

Marrone, D. P., Battat, J., Bensch, F., et al. 2004, ApJ, 612, 940

Minier, V., André, P., Bergman, P., et al. 2009, A\&A, 501, L1

Moisés, A. P., Damineli, A., Figuerêdo, E., et al. 2011, MNRAS, 411, 705

Mottram, J. C., Hoare, M. G., Davies, B., et al. 2011, ApJ, 730, L33

Ott, S. 2010, in Astronomical Data Analysis Software and Systems XIX, eds. Y. Mizumoto, K.-I. Morita, \& M. Ohishi, ASP Conf. Ser., 434, 139

Peng, T.-C., Wyrowski, F., Zapata, L. A., Güsten, R., \& Menten, K. M. 2012, A\&A, accepted

Phillips, T. G., Knapp, G. R., Wannier, P. G., et al. 1981, ApJ, 245, 512

Pilbratt, G. L., Riedinger, J. R., Passvogel, T., et al. 2010, A\&A, 518, L1

Roelfsema, P. R., Helmich, F. P., Teyssier, D., et al. 2012, A\&A, 537, A17
Rolffs, R., Schilke, P., Wyrowski, F, et al. 2011, A\&A, 527, A68

San José-García, I., Mottram, J. C., Kristensen, L. E., et al. 2012, A\&A, submitted

Schöier, F. L., van der Tak, F. F. S., van Dishoeck, E. F., \& Black, J. H. 2005, A\&A, 432, 369

Schuller, F., Menten, K. M., Contreras, Y., et al. 2009, A\&A, 504, 415

Snell, R. L., Scoville, N. Z., Sanders, D. B., \& Erickson, N. R. 1984, ApJ, 284, 176

Stutzki, J., \& Güsten, R. 1990, ApJ, 356, 513

Urquhart, J. S., Hoare, M. G., Lumsden, S. L., et al. 2011, MNRAS, 2112

van der Tak, F. F. S., Black, J. H., Schöier, F. L., Jansen, D. J., \& van Dishoeck, E. F. 2007, A\&A, 468, 627

van Dishoeck, E. F., Kristensen, L. E., Benz, A. O., et al. 2011, PASP, 123, 138

White, G. J., Abergel, A., Spencer, L., et al. 2010, A\&A, 518, L114

Wilson, T. L., \& Rood, R. 1994, ARA\&A, 32, 191

Wilson, T. L., Muders, D., Kramer, C., \& Henkel, C. 2001, ApJ, 557, 240

Wilson, T. L., Muders, D., Dumke, M., Henkel, C., \& Kawamura, J. H. 2011, ApJ, 728, 61

Wyrowski, F., Menten, K. M., Schilke, P., et al. 2006, A\&A, 454, L91

Wyrowski, F., Bergman, P., Menten, K., et al. 2008, Ap\&SS, 313, 69

Yang, B., Stancil, P. C., Balakrishnan, N., \& Forrey, R. C. 2010, ApJ, 718, 1062 